Commun. math. Phys. 7, 21--50 (1968)

\title{
On the Primitive Characters of the Poincaré Group
}

\author{
H. Joos and R. Schrader \\ Deutsches Elektronen Synchrotron Desy, Hamburg
}

Received June 15, 1967

\begin{abstract}
We calculate explicitely the traces of the different types of irreducible representations of the Poincaré group. These have the form of generalized class functions and their mathematical structure follows from a generalization to noncompact groups of Frobenius' construction of induced characters.
\end{abstract}

\section{Introduction}

Representation theory of the Poincaré group $\mathfrak{P}$ plays a fundamental role in relativistic kinematics of elementary particles [1]. Therefore it is natural to ask which of the well-known techniques of representation theory can be developped for this group. In this paper we study the problem of the characters of the irreducible representations of $\mathfrak{P}$.

The character $\chi(g)$ of a finite dimensional representation of a finite or compact group $G=\{g\}$ by linear transformations $T(g)$ is defined as

$$
\chi(g)=\operatorname{Trace} T(g) \text {. }
$$

Equivalent representations have the same characters. The characters $\chi^{m}(g)$ of irreducible representations are called primitive. The set of primitive characters $\left\{\chi^{m}(g)\right\}$ is called the dual space $\hat{G}$ of $G$.

The properties

and

$$
\chi\left(f g f^{-1}\right)=\chi(g) \quad f, g \in G
$$

$$
\chi(g f)=\chi(f g)
$$

respectively follow immediately. Functions with property (1.2) being constant on conjugation classes are called class functions.

In the theory of representations of finite or compact groups it is shown [2], that the primitive characters are idempotent with respect to convolution

$$
\int_{G} d \mu(f) \chi^{m}\left(g f^{-1}\right) \chi^{n}(f)=\frac{\delta_{m n} \chi^{m}(g)}{\operatorname{dim}(m)} .
$$

Here $\mathrm{d} \mu(f)$ denotes the normalized Haar measure of $G$, the dimension of the representation with character $\chi^{m}(g)$ is $\operatorname{dim}(m)$. Because every representation of a compact group is equivalent to a unitary one, for 
which $\bar{\chi}(g)=\chi\left(g^{-1}\right)$ holds, Equ. (1.4) implies the orthogonality relations .

$$
\int_{G} d \mu(f) \overline{\chi^{m}}(f) \chi^{n}(f)=\delta_{m n} .
$$

The primitive characters of such groups constitute a complete, orthogonal set in the space of class functions $\left\{\varphi(g) \mid \varphi\left(f g f^{-1}\right)=\varphi(g)\right\}$ :

$$
\varphi(g)=\sum_{m \in \hat{G}} \chi^{m}(g) \int_{G} d \mu(f) \overline{\chi^{m}}(f) \varphi(f) .
$$

The Poincaré group is a non-compact group and possesses only infinite dimensional faithful unitary representations. In general the traces of infinite dimensional unitary transformations are not defined, and the theory mentioned above cannot be applied. There is a theory of unitary, infinite dimensional representations of classical groups [3], in particular of the homogeneous Lorentz group, in which characters are given as generalized functions. Besides $\mathfrak{P}$ is not a classical group, we show in the following that we can define and calculate the primitive characters of the Poincaré group in a similar form [4]. So our discussion gives some impression of what could be expected about characters of non-compact, non-semisimple Lie groups.

A complete classification of the irreducible unitary representations of $\mathfrak{P}$ was first given by E. P. Wigner [1], supplemented by V. BARGMAN [5]. There are four main classes of representations

$$
\text { I. } P_{\mu} P^{\mu}>0 \text {, II. } P_{\mu} P^{\mu}=0 \text {, III. } P_{\mu} P^{\mu}<0 \text {, IV. } P_{\mu} \equiv 0
$$

where $P_{\mu}$ are the operators of the infinitesimal translations, i.e. the 4-momentum operators. Only the first two classes are considered as applicable in physics. We shall treat the complete problem, because we know from the treatment of compact groups, that a relation should exist between the structure of the conjugation classes of $\mathfrak{P}$ on the one hand and the set of all primitive characters on the other. The completeness relation reflects this connection.

G. W. Mackey [6] generalized Frobenius' induction procedure for the construction of unitary representations of separable, locally compact groups and thus developped a generalized mathematical theory of representations of such groups as $\mathfrak{P}$. By considering the irreducible representations of $\mathfrak{P}$ as induced representations we gain an immediate insight into the structure of the primitive characters of $\mathfrak{P}$, which corresponds to the induced characters of finite groups as discussed by Frobenius [7].

In the following chapter II we give some notations and a complete discussion of the classes of conjugate elements of the Poincaré group. Then we illustrate the problems involved by calculating the characters of the most important representations with $P_{\mu} P^{\mu}>0$, applying the 
usual non-rigorous methods of mathematical physics. In the same chapter III we give the results for the other cases. Chapter IV contains a mathematically more rigorous discussion of the problem.

\section{Conjugation Classes of the Poincaré Group}

The Poincaré group $\mathfrak{P}$ is the group of transformations $\{(\Lambda, a)\}$ between coordinates $x, x^{\prime}$ of inertial systems ${ }^{1}$

$$
x^{\prime}=\Lambda x+a \equiv(\Lambda, a) \cdot x .
$$

Here $\Lambda$ denotes a Lorentz transformation which we assume to be proper and orthochronous in the following. The group multiplication is defined by equ. (2.1) as

$$
\begin{gathered}
(\Lambda, a)\left(\Lambda^{\prime}, a^{\prime}\right)=\left(\Lambda \Lambda^{\prime}, a+\Lambda a^{\prime}\right) \\
(\Lambda, a)^{-1}=\left(\Lambda^{-1},-\Lambda a\right), \quad e=(1,0) .
\end{gathered}
$$

The Poincaré group is a semi-direct product of the homogeneous Lorentz group $\mathscr{L}_{+}^{T}=\{(\Lambda, 0)\}$ and the translation group $\mathfrak{T}=\{(1, a)\}$ with $\mathfrak{T}$ as invariant sub-group and $\mathscr{L}_{-}^{\mathbb{T}} \cong \mathfrak{P} / \mathfrak{T}$. Thus $\mathfrak{P}$ is a non-semisimple Lie group.

It is usual to consider, instead of $\mathfrak{P}$, its universal covering group $\overline{\mathfrak{P}}=\{(\alpha, a)\}$ with

$$
(\alpha, \alpha)\left(\alpha^{\prime}, \alpha^{\prime}\right)=\left(\alpha \alpha^{\prime}, \alpha+\Lambda_{\alpha} \alpha^{\prime}\right) \quad \text { etc. }
$$

Here $\alpha, \alpha^{\prime}$ denote unimodular, 2 -dimensional matrices

$$
\alpha=\left(\begin{array}{ll}
\alpha_{1}^{1} & \alpha_{1}^{2} \\
\alpha_{2}^{1} & \alpha_{2}^{2}
\end{array}\right) \in S L(2, \mathbb{C})
$$

and $\Lambda_{\alpha}$ is the homomorphism of $S L(2, \mathbb{C})$ on $\mathscr{L}_{+}^{\mathbb{T}}$. The group $S L(2, \mathbb{C})$ is a twofold covering group of $\mathscr{L}_{+}^{\top}$ with the kernel $\mathbb{R}=\{1,-1\}$. In the spinor calculus [8] we assign to every 4 -vector $x$ a hermitean matrix

$$
X=\left\{x_{0}+\mathbf{x} \boldsymbol{\sigma}\right\}
$$

by means of the well-known Pauli matrices $\boldsymbol{\sigma}$. Is $X^{\prime}$ the matrix of $\Lambda_{\alpha} x$, then $\Lambda_{\alpha}$ is explicitely given by

$$
X^{\prime}=\alpha X \alpha^{+} .
$$

Now we consider the classes of conjugate elements of $\overline{\mathfrak{P}}$. The element $(\alpha, a) \in \overline{\mathcal{P}}$ is conjugate to $\left(\alpha^{\prime}, a^{\prime}\right):(\alpha, a) \sim\left(\alpha^{\prime}, a^{\prime}\right)$ if and only if there is an element $(\beta, b)$ such that

$$
\begin{aligned}
\left(\alpha^{\prime}, a^{\prime}\right) & =(\beta, b)(\alpha, a)(\beta, b)^{-1} \\
& =\left(\beta \alpha \beta^{-1},\left(1-\Lambda_{\beta \alpha \beta^{-1}}\right) b+\Lambda_{\beta} a\right) .
\end{aligned}
$$

In order that $(\alpha, a) \sim\left(\alpha^{\prime}, a^{\prime}\right)$ in $\bar{P}$, it is necessary that $\alpha \sim \alpha^{\prime}$ in $S L(2, \mathbb{C})$. According to Jordan's normal form of $\alpha$ in $S L(2, \mathbb{C})$ we may distinguish

\footnotetext{
1 Our notation: 4 - vector $x=\left(x_{\mu}\right) \equiv\left(x_{0}, \mathbf{x}\right)$, invariant product $x y=x_{0} y_{0}$ $-x_{1} y_{1}-x_{2} y_{2}-x_{3} y_{3} \equiv x_{0} y_{0}-\mathbf{x y}$.
} 
the following types of conjugation classes:

$\mathrm{I}: \quad \alpha \sim \alpha_{\lambda}=\left(\begin{array}{ll}\lambda & 0 \\ 0 & \lambda^{-1}\end{array}\right), \quad \lambda=e^{1 / 2(\eta+i \varphi)}, \quad 0<\varphi<2 \pi, \quad 0<|\eta|<\infty$

II: $\quad \alpha \sim \alpha_{\lambda} ; \quad \varphi=0,2 \pi, \quad 0<\eta<+\infty$

III: $\quad \alpha \sim \alpha_{\lambda} ; \quad 0<\varphi<2 \pi, \quad \eta=0$

IV: $\quad \alpha= \pm 1$

$$
\mathrm{V}: \quad \alpha \sim \gamma_{ \pm}= \pm\left(\begin{array}{ll}
1 & 1 \\
0 & 1
\end{array}\right) \text {. }
$$

We want to construct a system of representatives of the conjugation classes of $\overline{\mathfrak{P}}$. We remark that if $\alpha$ is of type $\mathrm{I}$ then $\left(1-\Lambda_{\alpha}\right)$ has an inverse and we get

$$
\left(1,-\left(1-\Lambda_{\alpha}\right)^{-1} a\right)(\alpha, a)\left(1,-\left(1-\Lambda_{\alpha}\right)^{-1} a\right)^{-1}=(\alpha, 0) .
$$

In the case of $\alpha$ 's of types II or III the matrix

$$
E\left(\Lambda_{\alpha}\right)=\left(4-\operatorname{Trace} \Lambda_{\alpha}\right)^{-1}\left(\Lambda_{\alpha}-1\right)\left(\Lambda_{\alpha}^{-1}-1\right) \equiv E_{\alpha}
$$

describes the projection on the range of $\left(1-\Lambda_{\alpha}\right)$. Transforming with $(1, b), b=\left(4-\operatorname{Trace} \Lambda_{\alpha}\right)^{-1}\left(\Lambda_{\alpha}^{-1}-1\right) a$, gives

$$
(1, b)(\alpha, a)(1, b)^{-1}=\left(\alpha,\left(1-E_{\alpha}\right) a\right) .
$$

Further we may transform with a homogeneous Lorentz transformation which commutes with $\alpha$. We get

$(\beta, 0)\left(\alpha,\left(1-E_{\alpha}\right) a\right)(\beta, 0)^{-1}=\left(\alpha,\left(1-E_{\alpha}\right) \Lambda_{\beta} a\right)$ for $\beta \alpha \beta^{-1}=\alpha$.

For the type IV conjugation classes it follows immediately from (2.4) that $(+1, a) \sim\left(+1, a^{\prime}\right)$ if and only if there is a $\beta \in S L(2, \mathbb{C})$ such that $a=\Lambda_{\beta} a^{\prime}$.

In the case of $\alpha$ 's of type $\mathrm{V}, \Lambda_{\alpha}$ has an elementary divisor. According to E. P. Wigner [1] there is a base of 4 -vectors $\{u, v, w, z\}$ which is uniquely characterized by

and

$$
\Lambda_{\alpha} u=u, \quad \Lambda_{\alpha} v=v, \quad \Lambda_{\alpha} w=w+v, \quad \Lambda_{\alpha} z=z+w+\frac{1}{2} v
$$

$$
\begin{gathered}
(z, z)=(v, v)=(z, w)=(z, u)=(w, u)=(v, u)=(v, w)=0 \\
(z, v)=1, \quad(u, u)=(w, w)=-1, \quad z_{0}>0, \quad v_{0}>0 .
\end{gathered}
$$

We represent the translation vector $a$ in this base

$$
a=a_{u} u+a_{v} v+a_{w} w+a_{z} z
$$

and transform with $(1, b), b=\left(a_{v}-\frac{1}{2} a_{w}\right) w+a_{w} z$, then we get

$$
(1, b)(\alpha, a)(1,-b)=\left(\alpha, a_{u} u+a_{z} z\right) \text {. }
$$


For $a_{z} \neq 0$ we may now transform with $\left(\beta, b^{\prime}\right), \beta \alpha \beta^{-1}=\alpha$ and

with the result

$$
\begin{aligned}
& \Lambda_{\beta} u=u-\frac{a_{u}}{a_{z}} v, \quad \Lambda_{\beta} v=v, \quad \Lambda_{\beta} w=w \\
& \Lambda_{\beta} z=z-\frac{a_{u}}{a_{z}} u+\frac{1}{2}\left(\frac{a_{u}}{a_{z}}\right)^{2} v, \quad b^{\prime}=-\frac{1}{2} \cdot \frac{a_{u}^{2}}{a_{z}} w
\end{aligned}
$$

$$
\left(\beta, b^{\prime}\right)\left(\alpha, a_{u} u+a_{z} z\right)\left(\beta, b^{\prime}\right)^{-1}=\left(\alpha, a_{z} z\right) .
$$

For $a_{z}=0$ we can show that $a_{u}$ is invariant under conjugation.

Now we combine these remarks in order to show that every element of $\overline{\mathfrak{P}}$ is conjugate to one element of the following list:

$$
\begin{aligned}
& \text { I. } \quad\left(\alpha_{\lambda}, 0\right) \\
& \text { II. } \quad\left(\alpha_{\lambda},\left(0, a_{1}, 0,0\right)\right) \quad 0 \leqq a_{1}<\infty \\
& \text { III. (1) }\left(\alpha_{\lambda},\left(a_{0}, 0\right)\right) \quad-\infty<a_{0}<+\infty \\
& \text { (2) } \quad\left(\alpha_{\lambda},\left(0,0,0, a_{3}\right)\right) \quad 0<\left|a_{3}\right|<+\infty \\
& \text { (3) } \quad\left(\alpha_{\lambda},\left(\sigma_{0}, 0,0, \sigma_{1}\right)\right) \quad \sigma_{0}, \sigma_{1}= \pm 1 \\
& \text { IV. (1) (土1, } \left.\left(a_{0}, 0\right)\right) \quad-\infty<\alpha_{0}<+\infty \\
& \text { (2) } \quad\left( \pm 1,\left(0,0,0, a_{3}\right)\right) \quad 0<a_{3}<+\infty \\
& \text { (3) } \quad( \pm 1,( \pm 1,0,0,1)) \\
& \text { V. (1) } \quad\left(\gamma_{ \pm},\left(a_{0}, 0,0,-a_{0}\right)\right)-\infty<a_{0}<+\infty \\
& \text { (2) } \quad\left(\gamma_{ \pm},\left(0,0, a_{2}, 0\right)\right) \quad 0<\left|a_{2}\right|<+\infty \text {. }
\end{aligned}
$$

The $\alpha_{\lambda}$ satisfy the restrictions of the corresponding classes of Equ. (2.5).

Two elements of this list with different parameters belong to different conjugation classes.

In order to prove this statement we consider the most general class function on $\overline{\mathfrak{P}}$. For this we introduce several functions on $\overline{\mathfrak{P}}$ which correspond to the parameters of the representatives of the conjugation classes in the list above:

$$
\begin{aligned}
& \lambda(\alpha)=e^{1 / 2(\eta+i \varphi)}=\frac{1}{2}\left(\alpha_{1}^{1}+\alpha_{2}^{2} \pm \sqrt{\left.\left(\alpha_{1}^{1}+\alpha_{2}^{2}\right)^{2}-4\right)}\right. \\
& v^{2}(\alpha, a)=\left(\left(1-E_{\alpha}\right) a\right)^{2} \quad \text { defined for } \quad \lambda(\alpha) \neq \pm 1 \\
& =a^{2}-\frac{\left(\left(\Lambda_{\alpha}-1\right)\left(\Lambda_{\alpha}^{-1}-1\right) a\right)^{2}}{\left(4-\operatorname{Trace} \Lambda_{\alpha}\right)^{2}} \\
& c(\alpha, a)=\frac{1}{4}\left(\left(\Lambda_{\alpha}-1\right)^{2} a, a\right) \\
& d(\alpha, a)=a_{u} \quad \text { defined for } \lambda(\alpha)= \pm 1, \alpha \neq \pm 1 \text { in Equ. (2.11) } \\
& \sigma_{0}(\alpha, a)=\operatorname{sign} v_{0} \equiv \varepsilon\left(v_{0}\right), \quad v_{\mu}=\left(\left(1-E_{\alpha}\right) a\right)_{\mu} \\
& \sigma_{1}(\alpha, a)=\varepsilon\left(w_{0}\right) \varepsilon(\sin \varphi), \quad w_{\mu}=\varepsilon_{\mu}{ }^{\nu \rho \sigma}\left(\Lambda_{\alpha}\right)_{\varrho \sigma} a_{v} \\
& \sigma_{2}(\alpha, a)=\varepsilon\left(\left(\left(\Lambda_{\alpha}-1\right)^{2} a\right)_{0}\right) \\
& \sigma_{3}(\alpha, a)=\varepsilon\left(a_{u}\right) \quad \text { defined for } \lambda(\alpha)= \pm 1, \alpha \neq \pm 1 \text { in Equ. (2.11) (2.16) . }
\end{aligned}
$$


These functions have the following values for our representatives of list (2.15):

$\lambda(\alpha, a)=\lambda$ for classes of type I-IV

$\lambda\left(\gamma_{ \pm}, a\right)= \pm 1$ for classes of type $\mathrm{V}$

$v^{2}(\alpha, a)=-a_{1}^{2}$ for classes of type II

$v^{2}(\alpha, a)=a_{0}^{2}, \quad \sigma_{0}(\alpha, a)=\varepsilon\left(a_{0}\right) \quad$ for classes of type (III) 1

$v^{2}(\alpha, a)=-a_{3}^{2}, \sigma_{1}(\alpha, a)=-\varepsilon\left(a_{3}\right)$ for classes of type III (2)

$c(\alpha, a)=a_{0}^{2}, \quad \sigma_{2}(\alpha, a)=\varepsilon\left(a_{0}\right) \quad$ for classes of type $\mathrm{V}(1)$

$d(\alpha, a)=a_{2}^{2}, \quad \sigma_{3}(\alpha, a)=\varepsilon\left(a_{2}\right) \quad$ for classes of type $\mathrm{V}(2)$

$\sigma_{0}(\alpha, a)=\sigma_{0}, \quad \sigma_{1}(\alpha, a)=\sigma_{1} \quad$ for classes of type III $(3)$.

We note that the topological structure of the conjugation classes of $\overline{\mathfrak{P}}$ is quite involved. Lower dimensional sub-sets, e.g. in the $\lambda$ plane, are important. Therefore it is essential to discuss not only class functions on $\overline{\mathfrak{P}}$ but also generalized functions i.e. distributions on $\overline{\mathfrak{P}}$ which are invariant under conjugation. The generalized function of the form

$$
\begin{aligned}
F(\alpha, a)= & F_{I}(\lambda)+F_{I I, 1}\left(\eta, v^{2}\right) \Theta\left(-v^{2}\right) \delta(\varphi) \\
& +F_{\mathrm{II}, 1}^{\prime}\left(\eta, v^{2}\right) \Theta\left(-v^{2}\right) \delta(\varphi+2 \pi) \\
& +F_{\mathrm{III}, 1}\left(\varphi, v^{2}, \sigma_{0}\right) \Theta\left(v^{2}\right) \delta(\eta)+F_{\mathrm{III}, 2}\left(\varphi, v^{2}, \sigma_{1}\right) \Theta\left(-v^{2}\right) \delta(\eta) \\
& +F_{\mathrm{III}, 3}\left(\varphi, \sigma_{0}, \sigma_{1}\right) \delta\left(v^{2}\right) \delta(\eta) \\
& +\left\{F_{\mathrm{IV}, 1}\left(a^{2}, \varepsilon\left(a_{0}\right)\right) \Theta\left(a^{2}\right)+F_{\mathrm{IV}, 2}\left(a^{2}\right) \Theta\left(-a^{2}\right)\right. \\
& \left.+F_{\mathrm{IV}, 3}\left(\varepsilon\left(a_{0}\right)\right) \delta\left(a^{2}\right)\right\} \delta^{6}\left(\Lambda_{\alpha}-1\right) \\
& +\sum_{n=0,1}\left(F_{\mathrm{V}_{1}}\left(c, \sigma_{2}, n\right)+F_{\mathrm{V}, 2}\left(d, \sigma_{3}, n\right) \delta(c)\right) \delta(\eta) \delta(\varphi-2 \pi n)
\end{aligned}
$$

- where $\lambda, \varphi, \eta, v^{2}, c, d$, etc. denote the functions on $\overline{\mathfrak{P}}$ defined in (2.17) -, is invariant under conjugations in $\overline{\mathfrak{P}}$. This may easily be verified by direct calculations.

Two elements of the system of representatives (2.15) differ, according to $(2.17)$, in at least one of the parameters $\varphi, \eta, v^{2}, \ldots$ on the support of $F(\alpha, a)$ in (2.18). Therefore different elements of the list (2.15) represent different classes. Also, in a vague sense, (2.18) gives the most general generalized class function. We expect that the primitive characters of $\overline{\mathfrak{P}}$ have the form $(2.18)$.

\section{Elementary Calculation of the Primitive Characters}

In this chapter we calculate the traces of the unitary representations of $\overline{\mathfrak{P}}$ in a simple-minded and direct way. 
1. The Characters of the Representations with Time-Like Momentum $P_{\mu}$

The representations of $\overline{\mathfrak{P}}$ with mass $P_{\mu} P^{\mu}=M^{2}>0$, spin $s$ and energy $\operatorname{sign} \varepsilon_{1}=\operatorname{sign} P_{0}$ were given by E. P. WIGNER [1] as unitary transformations of the Hilbert space $\mathscr{H}$ of vector valued functions $\psi_{n}(p), \quad n=-s,-s+1, \ldots, s$, on the mass hyperboloid $p^{2}=M^{2}$, $\operatorname{sign} p_{0}=\varepsilon_{1}$, with the norm

$$
\|\psi\|^{2}=\sum_{n} \int\left|\psi_{n}(p)\right|^{2} \frac{d \mathbf{p}}{2\left|p_{0}\right|} .
$$

The representation $U(\alpha, a)$ is defined as

$$
(U(\alpha, a) \psi)_{n}(p)=\sum_{n^{\prime}} e^{i p a} D_{n n^{\prime}}^{s}(\varrho(\alpha, p)) \psi_{n^{\prime}}\left(\Lambda_{\alpha}^{-1} p\right) .
$$

The following definitions are used:

$$
\begin{aligned}
\varrho(\alpha, p) & =h(p) \alpha h^{-1}\left(\Lambda_{\alpha}^{-1} p\right) \in S U(2) \\
h(p) & =\left(2 M\left(M+\left|p_{0}\right|\right)\right)^{-1 / 2}\left\{p_{0}+M-\mathbf{p} \boldsymbol{\sigma}\right\}
\end{aligned}
$$

$\Lambda_{h(p)}$ has the property

$$
\Lambda_{h(p)} p=\left(\varepsilon_{1} M, \mathbf{0}\right) .
$$

$D_{n n^{\prime}}^{s}(\varrho)$ is the representation matrix of the $(2 s+1)$-dimensional irreducible representation of $S U(2)$ [5].

In order to evaluate the trace of $U(\alpha, a)$ we introduce in $\mathscr{H}$ an improper base $\left\{\Phi_{p, n}\right\}$ of eigenvectors of the infinitesimal translation operators $P_{\mu}$, which transform like

$$
U(\alpha, a) \Phi_{p, n}=\sum_{n^{\prime}} e^{i\left(\Lambda_{\alpha} p, a\right)} \Phi_{\Lambda_{\alpha} p, n^{\prime}} D_{n^{\prime} n}^{s}\left(\varrho\left(\alpha, \Lambda_{\alpha} p\right)\right)
$$

and which are normalized according to

$$
\left(\Phi_{p, n}, \Phi_{p^{\prime}, n^{\prime}}\right)=2\left|p_{0}\right| \delta\left(\mathbf{p}-\mathbf{p}^{\prime}\right) .
$$

We may consider the functions $\psi_{n}(p)$ as the coefficients of an arbitrary vector of $\mathscr{H}$ in this base.

Now we regard as the trace of $U(\alpha, a)$

$$
\text { Trace } U(\alpha, a)=\sum_{n} \int \frac{d^{3} p}{2\left|p_{0}\right|} \cdot\left(\Phi_{p, n}, U(\alpha, a) \Phi_{p, n}\right) .
$$

We are aware of the shortcomings of this definition. Unitary operators in an infinite dimensional Hilbert space do not posses a trace in the usual sense. Further one has to be careful in the use of an inproper base. Nevertheless we shall calculate (3.7) according to the usual methods of mathematical physics, leaving the justification of this procedure to the following chapter. 
With the help of Eqs. (3.5) and (3.6) we get with

$$
\text { Trace } U(\alpha, a)=\int d \mathbf{p} \cdot e^{i p a} \chi^{s}\left(h(p) \alpha h^{-1}(p)\right) \delta^{3}\left(\mathbf{p}-\boldsymbol{\Lambda}_{\alpha} \mathbf{p}\right)
$$

$$
\chi^{s}(\varrho)=\sum_{n} D_{n n}^{s}(\varrho)=\frac{\sin (2 s+1) \varphi / 2}{\sin \varphi / 2}
$$

the character of the representation $D_{n n^{\prime}}^{s}(\varrho)$ of $S U(2)$ [8].

From Eq. (3.8) we see that Trace $U(\alpha, a)$ is only different from zero if $\Lambda_{\alpha}$ leaves an time-like vector $p$ invariant, i.e. if $\alpha$ is conjugate in $S L(2, \mathbb{C})$ to an element of $S U(2)$; in this case $h(p)$ transforms $\alpha$ into a rotation $\varrho(\alpha, p)=h(p) \alpha h^{-1}(p)$. $\varrho(\alpha, p)$ is conjugate to $\varrho\left(\alpha, p^{\prime}\right)$ in $S L(2, \mathbb{C}): \varrho(\alpha, p)=h(p) h^{-1}\left(p^{\prime}\right) \varrho\left(\alpha, p^{\prime}\right) h\left(p^{\prime}\right) h^{-1}(p)$. But two elements of $S U(2)$ which are conjugate in $S L(2, \mathbb{C})$ correspond to rotations with the same rotation angle, as we may see from the remarks on Eq. (2.5). Therefore they are also conjugate to each other in $S U(2)$. Since $\chi^{s}(\varrho)$ is a class function on $S U(2)$, we may consider $\chi^{s}(\varrho(\alpha, p))$ in Eq. (3.8) as independent of $p$. We may put it in front of the integral as a factor $\chi^{s}(\varphi(\alpha))$ with $\varphi(\alpha)$ defined in Eq. (2.16).

Now we have to evaluate the integral $\int d \mathbf{p} e^{i p a} \delta^{3}\left(\mathbf{p}-\Lambda_{\alpha} \mathbf{p}\right)$ which is a class function of $(\alpha, a)$ in $S L(2, \mathbb{C})$. This follows in general from the class function property of the trace or it may be shown directly by substituting the integration variable $p^{\prime}=\Lambda_{\beta} p$ :

$$
\begin{aligned}
\int \frac{d \mathbf{p}}{2\left|p_{0}\right|} 2\left|p_{0}\right| \delta(\mathbf{p} & \left.-\Lambda_{\alpha} \mathbf{p}\right) e^{i p a} \\
& =\int \frac{d \mathbf{p}^{\prime}}{2\left|\mathbf{p}_{0}^{\prime}\right|} \cdot 2\left|p_{0}^{\prime}\right| \delta\left(\mathbf{p}^{\prime}-\Lambda_{\boldsymbol{\beta} \alpha \boldsymbol{\beta}^{-1}} \mathbf{p}^{\prime}\right) e^{i\left(p^{\prime}, \Lambda_{\beta} a\right)} .
\end{aligned}
$$

In the integral we put $\alpha=\alpha_{\lambda}$ and calculate

$$
\delta^{3}\left(\mathbf{p}-\Lambda \alpha_{\lambda} \mathbf{p}\right)=\frac{1}{4 \sin ^{2}(\varphi / 2)} \frac{1}{p_{0}} \delta\left(p_{1}\right) \delta\left(p_{2}\right) \delta(\eta) \quad p_{0}=+\sqrt{M^{2}+p_{3}^{2}}
$$

Thus expression (3.8) becomes

$$
\operatorname{Trace} U\left(\alpha_{2}, a\right)=\frac{\delta(\eta)}{4 \sin ^{2}(\varphi / 2)} \chi^{s}(\varphi) \int_{-\infty}^{+\infty} \frac{d p_{3}}{p_{0}} e^{i\left(p_{0} a_{0}-p_{3} a_{3}\right)}
$$

The remaining integral in (3.12) is the wellknown invariant function in 2 dimensions [9]

$$
\begin{aligned}
\Delta_{2}^{(\varepsilon)}\left(v ; M^{2}\right) & =\iint d p_{0} d p_{3} \Theta\left(\varepsilon p_{0}\right) \delta\left(p_{0}^{2}-p_{3}^{2}-M^{2}\right) e^{i\left(p_{0} a_{0}-p_{3} a_{3}\right)} \\
& =i \pi \cdot H_{0}^{(1)}\left(M \sqrt{\left(a_{0}^{2}-a_{3}^{2}\right)_{\varepsilon, \sigma_{0}}}\right) \\
v & =\left(a_{0}, a_{3}\right), \quad \sigma_{0}=\operatorname{sign} a_{0} .
\end{aligned}
$$

$H_{0}^{(1)}(z)$ is the Hankel function of the first kind [10] defined as an analytic function in the complex plane with the cut at $-\infty<z<0$. The cut of the square root runs along the positive axis, the $\pm \operatorname{sign}$ in $\sqrt{v_{ \pm}^{2}}$ indicates 
the approach from the upper or lower half plane. We have therefore

$$
\begin{gathered}
H_{0}^{(1)}\left(\sqrt{v_{\varepsilon}^{2}}\right)=H_{0}^{(1)}(|v|) \text { for } v^{2}>0, \varepsilon=+1 ;-H_{0}^{(2)}(|v|) \text { for } v^{2}>0, \varepsilon=-1 \\
\frac{2}{i \pi} K_{0}(|v|) \text { for } v^{2}<0, \varepsilon= \pm 1
\end{gathered}
$$

Now we remember the class function property of Trace $U(\alpha, a)$ which we have explicitly confirmed for the improper trace by calculation (3.10) and we get as final result the primitive characters of the unitary representations of $\overline{\mathfrak{P}}$ with time-like momentum $P_{\mu} P^{\mu}=M^{2}$, spin $s$ and energy $\operatorname{sign} \varepsilon_{1}$ :

$$
\chi\left(\alpha, a ; M^{2}, s, \varepsilon_{1}\right)=\frac{i \pi}{4 \sin ^{2}(\varphi / 2)} \delta(\eta) \chi^{s}(\varphi) H_{0}^{(1)}\left(M \sqrt{v_{\varepsilon_{1} \sigma_{n}}^{2}}\right)
$$

The arguments of the different functions are defined as in eq. (2.16). The primitive characters of type I representations of $\overline{\mathfrak{P}}$ are indeed class distributions of the form (2.18) with support on type III classes of conjugate elements.

\section{The Characters of the Representations with Light-Like Momentum $P_{\mu}$}

In the preceding section we have performed quite explicitly the calculation of the traces of the in physics most important representations of $\overline{\mathfrak{P}}$ of class I. Since the constructions of the class I, II, III representations of $\overline{\mathfrak{P}}$ have many features in common, we can be shorter in the calculations of the traces of the other representations. E. P. Wigner has constructed all the faithful irreducible unitary representations of $\overline{\mathfrak{P}}$ as transformations of vector-valued functions on a "momentum hypersurface" $\mathfrak{Z}$ which have the form of Eq. (3.2). In the general case the matrices $D^{s}(\varrho)$ form a representation of type $s$ of Wigner's "Little Group" $S_{i} \subset S L(2, \mathbb{C})$, i.e. the sub-group of $\overline{\mathfrak{P}}$ which leaves a fixed $p_{i} \in \mathfrak{M}$ invariant; $h_{i}(p)$ denotes any fixed choice of Lorentz transformations which has the property $\Lambda_{h_{i}(p)} p=p_{i}$ and it is $\varrho(\alpha, p)=h_{i}(p) \alpha h_{i}^{-1}\left(\Lambda_{\alpha}^{-1} p\right)$ as in Eq. (3.3). Therefore the characters of the type I, II, III representations of $\overline{\mathfrak{P}}$ will in general have the form of Eq. (3.8), with $\chi^{s}(\varrho)$ denoting the character of the representation $D^{s}(\varrho)$ of $S_{i}$, and with the integration being performed over the momentum hypersurface $\mathfrak{M}$. In the following we want to calculate this integral for type II representations.

The little group of the hyper-surface of light-like momenta $p^{2}=0$ is formed by the matrices

$$
\gamma(\varphi, u)=\left(\begin{array}{ll}
e^{i \varphi / 2}, & u e^{-i \varphi / 2} \\
0, & e^{-i \varphi / 2}
\end{array}\right) \in S_{\gamma} \quad u \in \mathbb{C}, \quad 0 \leqq \varphi<4 \pi .
$$

The corresponding Lorentz transformations leave the vector $p_{i}= \pm(1,0,0,1)$ invariant $\Lambda_{\gamma(\varphi, u)} p_{i}=p_{i}$. The group $S_{\gamma}$ may be re- 
garded as a twofold covering group of the Euclidean group in two dimensions [1]. This is indicated by the composition law:

$$
\gamma(\varphi, u) \gamma\left(\varphi^{\prime}, u^{\prime}\right)=\gamma\left(\varphi+\varphi^{\prime}, u+e^{i \varphi} u^{\prime}\right) .
$$

In order to find the conjugation classes of $S_{\gamma}$ we calculate

$$
\gamma\left(\varphi^{\prime}, u^{\prime}\right) \gamma(\varphi, u) \gamma^{-1}\left(\varphi^{\prime}, u^{\prime}\right)=\gamma\left(\varphi,\left(1-e^{i \varphi}\right) u^{\prime}+e^{i \varphi^{\prime}} u\right) .
$$

From this we see

$$
\begin{aligned}
& \gamma(\varphi, u) \sim \gamma(\varphi, 0) \text { in } S_{\gamma} \text { for } \varphi \neq 0,2 \pi \\
& \gamma(o, u) \sim \gamma(o,|u|), \quad \gamma(2 \pi, u) \sim \gamma(2 \pi,|u|) \text { in } S_{\gamma} .
\end{aligned}
$$

Obviously $\gamma(\varphi, 0), \varphi \neq 0,2 \pi$, and $\gamma(0, r), \gamma(2 \pi, r), r \geqq 0$ form a complete list of representatives of the conjugation classes of $S_{\gamma}$.

There are two classes of irreducible unitary representations of $S_{\gamma}[1]$ :

A. The 1-dimensional, unfaithful representations

$$
\gamma(\varphi, u) \rightarrow e^{i s \varphi}, \quad s=0 \pm 1 / 2, \pm 1, \ldots
$$

which are identical with their characters $\chi^{s}(\gamma)=e^{i s \varphi}$.

B. The representations in the function space $\mathscr{H}=\{\psi(v)\}$ with the norm

$$
\begin{gathered}
\|\psi(v)\|^{2}=\int_{e}^{2 \pi}|\psi(v)|^{2} \frac{d v}{2 \pi}, \quad 0 \leqq \nu \leqq 2 \pi: \\
(U(\gamma) \psi)(v)=(-1)^{\tau|\varphi, v|} \exp \left(i Q \operatorname{Re}\left(e^{i v} u\right) \psi(\nu+\varphi-2 \pi|\varphi, v|)\right.
\end{gathered}
$$

with $|\varphi, \nu|=0$ for $0 \leqq \nu+\varphi<2 \pi,|\varphi, \nu|=1$ for $2 \pi \leqq \nu+\varphi<4 \pi$ and $|\varphi, v|=2$ for $4 \pi \leqq \nu+\varphi<6 \pi$.

The parameters $Q, 0<Q<+\infty$, and $\tau=0,1$ distinguish different inequivalent representations. We have calculated the improper traces of this infinite dimensional representations in Appendix A with the result

$$
\text { Trace } U(\gamma)=\left\{\delta(\varphi)+(-1)^{\tau} \delta(\varphi-2 \pi)\right\} 2 \pi J_{0}(Q|u|) \text {. }
$$

We see that the characters are distributions in the parameters of the conjugation classes of $S_{\gamma}$ as introduced in (3.18). With the help of the formula [10]

$$
\int_{0}^{\infty} J_{0}(p x) J_{0}\left(p, x^{\prime}\right) p d p=\frac{1}{x} \delta\left(x-x^{\prime}\right)
$$

we see that the characters (3.19) and (3.21) form a complete orthogonal system of functions on the conjugation classes (3.18).

The explicit description of the class II representations of $\overline{\mathfrak{P}}$ may be given in the Hilbert space $\mathscr{H}$ of all complex valued functions $\psi(p)$ defined on the light cone $p^{2}=0, \varepsilon\left(p_{0}\right)=\varepsilon_{2}$, which are square integrable with 
respect to the measure $d \mu(p)=\frac{1}{2\left|p_{0}\right|} d^{3} p$. We use the following definitions

$$
\begin{aligned}
\varrho(\alpha, p) & =t(p) \alpha t^{-1}\left(\Lambda_{\alpha}^{-1} p\right) \equiv \gamma(\varphi(\alpha, p), u(\alpha, p)) \in S_{\gamma} \\
t(p) & =\exp \left(-\frac{\tau}{2} \sigma_{3}\right) \exp \left(-\frac{i}{2} \vartheta \sigma_{2}\right) \exp \left(-\frac{i}{2} \psi \sigma_{3}\right) \\
\tau & =\log |\mathbf{p}|, \quad(\vartheta, \psi) \text { the polar angles of } \varepsilon_{2} \mathbf{p} .
\end{aligned}
$$

$t(p)$ has similar properties as $h(p)$ :

$$
\Lambda_{t(\varrho)} p=\varepsilon\left(p_{0}\right)(1,0,0,1) \text {. }
$$

Then the representation A (3.19) of $S_{\gamma}$ give the following representation of $\overline{\mathfrak{P}}$ :

$$
(U(\alpha, \alpha) \psi)(p)=e^{i p a+i s \varphi(\alpha, p)} \psi\left(\Lambda_{\alpha}^{-1} p\right) .
$$

Defining the trace in exactly the same way as in (3.7), we obtain:

$$
\text { Trace } U(\alpha, a)=\int_{\varepsilon\left(\varrho_{0}\right)=\varepsilon_{2}} e^{i p a+i s \varphi(\alpha, p)} \delta\left(\mathbf{p}-\boldsymbol{\Lambda}_{\alpha} p\right) d^{3} p .
$$

We choose $\alpha=\alpha_{\lambda}$ and with $\varphi=\varphi(\alpha)$ according to (2.16) we get

$\operatorname{Trace} U\left(\alpha_{\lambda}, a\right)=\frac{\delta(\eta)}{4 \sin ^{2} \varphi / 2} \chi\left(\varphi, a_{0}, a_{3}\right)$
$\chi\left(\varphi, a_{0}, a_{3}\right)=e^{i s \varphi} \int_{0}^{\infty} \frac{d p}{p} e^{i \varepsilon_{2} p a_{-}}+e^{-i s \varphi} \int_{0}^{\infty} \frac{d p}{p} e^{-i \varepsilon_{2} p a_{+}}$

The integral

$$
\int_{0}^{\infty} \frac{d p}{p} e^{i p x}=-i \frac{\pi}{2} \operatorname{sign} x-\log |x|-C
$$

determines a distribution only up to a constant, i.e. a distribution on a hyperplane of test functions defined by $\int \varphi(x) d x=0$. Therefore our simple minded calculation of the characters becomes somewhat arbitrary in this case. We refer to our discussion in section IV. In our evaluation we use the fact that Trace $U(\alpha, a)$ must be a class-function. We get as a result:

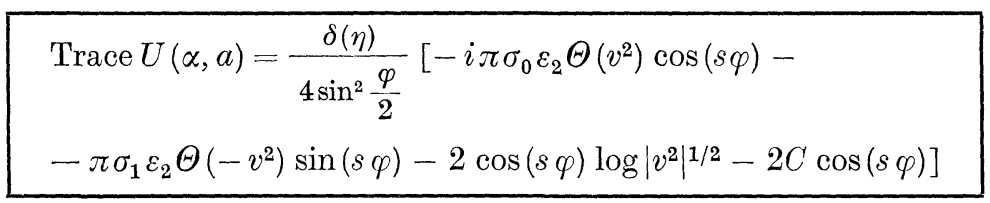

which defines the characters of the unitary representations of $\overline{\mathcal{F}}$ with lightlike momentum $P_{\mu} P^{\mu}=0$, "spin" $s$ and energy sign $\varepsilon_{2}$. The meaning of the arbitrary constant $C$ will become clear below.

We want to add a remark on the limit $M^{2} \rightarrow 0$ of the characters of $\overline{\mathfrak{P}}$ with timelike momentum $P^{\mu} P_{\mu}=M^{2}$, spin $s$ and energy sign $\varepsilon_{1}$. We 
may compare them with the characters of lightlike momentum: For small $x, H_{0}^{(1)}(x)$ has the expansion [10]

$$
H_{0}^{(1)}(x)=1+\frac{2 i}{\pi}\left(\gamma+\log \frac{x}{2}\right)+\ldots
$$

$\gamma$ being the Euler constant.

Up to a constant, we therefore obtain approximately

$$
H_{0}^{(1)}\left(M \sqrt{v_{\varepsilon_{1}}^{2}, \sigma_{0}}\right)=\frac{2 i}{\pi}\left(\log \left|\sqrt{v^{2}}\right|-\frac{\pi i}{2} \varepsilon_{1} \sigma_{0} \Theta\left(v^{2}\right)\right)
$$

for small $M ; H_{0}^{(1)}$ regarded as a function of $v^{2}, \varepsilon_{1}, \sigma_{0}$. Comparing (3.29) and (3.14) we thus get the following "helicity-decomposition" [11] of the characters with timelike momentum:

$\lim _{M^{2} \rightarrow 0} \operatorname{Trace} U\left(\alpha, a ; M^{2}, s, \varepsilon_{1}\right)=\sum_{s^{\prime}=-s}^{s^{\prime}=s} \operatorname{Trace} U\left(\alpha, a ; P_{\mu} P^{\mu}=0, s^{\prime}, \varepsilon_{1}\right)$.

The representations B (3.20) of $S_{\gamma}$ give the following representations of $q \mathcal{j}$ $(U(\alpha, a) \psi)(v, p)=$

$(-1)^{\tau|\varphi(\alpha, p), \nu|} \exp \left(i Q R e\left(e^{i v} u(\alpha, a)\right) \psi\left(\nu+\varphi(\alpha, \varrho)-2 \pi|\varphi, \nu|, \Lambda_{\alpha}^{-1} p\right)\right.$

with notations as in $(3.23) \ldots(3.25)$.

We were not able to calculate in this formal manner the characters of those representations of $\overline{\mathfrak{P}}$ which are given by the representations (3.20) of $S_{\gamma}$. The difficulty in calculating the characters of these representations is due to the fact that their support must be contained in the set $V$ of (2.15) Chap. II, and which seems to be too "small". However, we are able to determine the character as distribution in the framework of our mathematical theory in chapter IV.

\section{The Characters of the Representations with Space-Like Momentum}

The little group of the hypersurface of space-like momenta $p^{2}=-\hat{M}^{2}<0$ is formed by the matrices of the form

$$
\beta=\left(\begin{array}{ll}
x & y \\
\bar{y} & \bar{x}
\end{array}\right) \in S, \quad \operatorname{det} \beta=1 .
$$

The corresponding Lorentz transformations now leave the vector $p_{i}=(0,0,0,1)$ invariant. $S_{\beta}$ is the group of linear transformations, which map the interior of the unit circle onto itself. $S_{\gamma}$ is isomorphic to $S L(2, \mathbb{R})$.

A complete list of representatives of the conjugation classes of $S_{\beta}$ is given by

$$
\begin{aligned}
& C_{\beta}^{1}: \xi\left(\begin{array}{ll}
\cosh \frac{\eta}{2} & \sinh \frac{\eta}{2} \\
\sinh \frac{\eta}{2} & \cosh \frac{\eta}{2}
\end{array}\right), \quad C_{\beta}^{2}:\left(\begin{array}{ll}
e^{i \varphi / 2} & 0 \\
0 & e^{-i \varphi / 2}
\end{array}\right), \quad C_{\beta}^{3}: \xi\left(\begin{array}{rr}
1+i & i \\
-i & 1-i
\end{array}\right) \\
& \xi= \pm 1, \quad \eta>0 \quad 0 \leqq \varphi<4 \pi \quad \xi= \pm 1 \text {. }
\end{aligned}
$$


We shall consider only those irreducible representations of $S_{\beta}$ which appear in the Plancherel formula for $S_{\beta} \cong S L(2, \mathbb{R})$. They are given, according to Bargmann [5], by the Main Series and the Discrete Series:

A. The Main Series

The representation is given by the unitary transformations $D(\beta)$ of the Hilbert space $\mathscr{H}=\{\psi(\varphi)\},\|\psi\|^{2}=\frac{1}{2 \pi} \int_{-\pi}^{+\pi}|\psi(\varphi)|^{2} d \varphi$ :

with

$$
(D(\beta) \psi)(\varphi)=\mu\left(\beta, \beta^{-1} \varphi\right) \psi\left(\beta^{-1} \varphi\right)
$$

and

$$
e^{-i \beta^{-1} \varphi}=\left(x e^{i \varphi}-y\right) /\left(\bar{x}-\bar{y} e^{i \varphi}\right)
$$

$\mu(\beta, \varphi)=\left(x+e^{i \varphi} y\right)^{\tau_{1}}\left|x+e^{i \varphi} y\right|^{-1-\tau_{1}+i \sigma}, 0<\sigma<\infty, \tau_{1}=0,1 .(3.36)$

$B$. The Discrete Series

The representation space is given by the linear space of all functions, which are analytic in the interior of the unit circle:

a) $(D(\beta) \psi)(z)=\mu\left(\beta, \beta^{-1} z\right)^{2 x} \psi\left(\beta^{-1} z\right)$

b) $(D(\beta) \psi)(z)=\overline{\mu\left(\beta, \beta^{-1} \bar{z}\right)^{-2 x}} \psi \overline{\left(\beta^{-1} z\right)}$

with

$$
\begin{gathered}
\mu(\beta, z)=x+y z, \quad \beta z=\frac{\bar{x} z+\bar{y}}{y z+x}, 2 \varkappa=1,2,3, \ldots, \quad z=u+i v \\
\|\psi\|_{\varkappa}^{2}=\frac{(2 \varkappa-1)}{\pi} \int_{|z|<1}(1-z \bar{z})^{2 \varkappa-2} \overline{\psi(z)} \psi(z) d u d v \quad 2 \varkappa \neq 1
\end{gathered}
$$

and

$$
\|\psi\|_{1 / 2}^{2}=\lim _{2 x \rightarrow 1} \frac{(2 x-1)}{\pi} \int_{|z|<1}(1-z \bar{z})^{2 x-2} \overline{\psi(z)} \psi(z) d u d v
$$

We distinguish the cases a) and b) by $\tau_{2}$ :

$$
\tau_{2}=1 \text { for case a), } \tau_{2}=-1 \text { for case b) . }
$$

The improper traces of these representations are [12]:

$$
\begin{aligned}
& \text { a) } \operatorname{Trace} D(\beta)=( \pm 1)^{\tau_{1}} \frac{\cos \sigma \frac{\eta}{2}}{\sinh \frac{\eta}{2}} \text { for } \beta \in C_{\beta}^{1} \\
& =0 \quad \text { for } \quad \beta \in C_{\beta}^{2}, C_{\beta}^{3}
\end{aligned}
$$

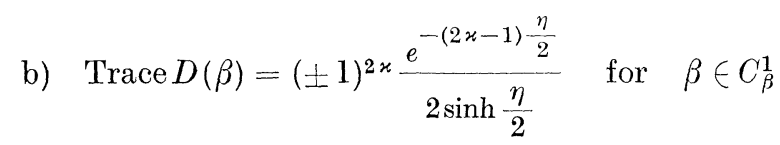

$$
\begin{aligned}
& =i(-1)^{\frac{1-\tau_{2}}{2}} \frac{e^{i(2 x-1)}}{2 \sin \frac{\varphi}{2}} \text { for } \beta \in C_{\beta}^{2} .
\end{aligned}
$$


In order to construct the type III representations of $\overline{\mathfrak{P}}$, consider the set of all vector-valued functions $\psi(p)$ on the spacelike hypersurface $p^{2}=-\widehat{M}^{2}<0$ where the values are taken in one of the representation spaces of (3.36) or (3.37), such that

$$
\|\psi\|^{2}=\int_{p^{2}=-\hat{M}^{2}}(\psi(p), \psi(p)) \cdot\left(p_{0}^{2}+\hat{M}^{2}\right)^{1 / 2} \frac{d p_{0}}{2} d \Omega_{\hat{\mathbf{p}}}<\infty
$$

$d \Omega_{\mathbf{p}}$ is the differential solid angle of $\hat{\mathbf{p}}=\frac{\mathbf{p}}{|\mathbf{p}|}$ and $(\psi(p), \psi(p))$ is the scalar product in the representation space of (3.36) or (3.37). This set defines a complex Hilbert space.

We define

$$
\begin{aligned}
r(\gamma, \delta, \varepsilon) & =\left(\gamma_{0}+\gamma \sigma_{3}\right)\left(\delta_{0}+i \delta \sigma_{2}\right)\left(\varepsilon_{0}+i \varepsilon \sigma_{3}\right) \\
\gamma_{0}^{2}-\gamma^{2} & =\delta_{0}^{2}+\delta^{2}=\varepsilon_{0}^{2}+\varepsilon^{2}=1
\end{aligned}
$$

then the equation

$$
\left(\Lambda_{r^{-1}(\gamma, \delta, \varepsilon)} p\right)=(0,0,0, \hat{M})
$$

defines a correspondence $r(\gamma, \delta, \varepsilon) \leftrightarrow p$ in a one-to-one-way, except on a set of measure zero on the spacelike hypersurface. Setting

$$
\varrho(\alpha, p)=r(p) \alpha r^{-1}\left(\Lambda_{\alpha}^{-1} p\right) \in S_{\beta}
$$

we obtain an irreducible representation of $\overline{\mathfrak{P}}$ on the Hilbert space defined above:

$$
(U(\alpha, a) \psi)(p)=e^{i p a} D(\varrho(\alpha, p)) \psi\left(\Lambda_{\alpha}^{-1} p\right)
$$

where $D$ is given by (3.36) and (3.37).

Taking what has become the standard definition of Trace, we obtain Trace $U(\alpha, a)$

$=\int_{p^{2}}=-M^{2} e^{i p a} \operatorname{Trace} D(\varrho(\alpha, p)) \delta\left(p_{0}-\left(\Lambda_{\alpha} p\right)_{0}\right) \delta\left(\Omega_{\mathbf{p}}-\Omega_{\Lambda_{\alpha \mathbf{p}}}\right) d p_{0} d \Omega_{\hat{\mathbf{p}}}$

Again we notice that Trace $U(\alpha, a)$ is only different from zero if $\alpha$ possesses a spacelike eigenvector. This is exactly the case if $\alpha \sim \alpha_{\lambda}$, $\alpha_{\lambda} \in$ II, III, IV or V in (2.5). We calculate only the first two types II and III. If $\alpha_{\lambda}$ is of type II, elements $\varrho(\alpha, p)$ which are conjugate under $S L(2, \mathbb{C})$ are also conjugate under $S_{\beta}$. In that case we may consider Trace $D(\varrho(\alpha, p))$ in $(3.45)$ as independent of $p$. For the Main series we therefore obtain:

$$
\begin{aligned}
& \text { Trace } U(\alpha, a) \\
& =\frac{\left(\delta(\varphi)+(-1)^{\tau_{1}} \delta(\varphi-2 \pi)\right)}{4 \sinh ^{3} \frac{\eta}{2}} \cos \sigma \frac{\eta}{2} \cdot 2 \pi J_{0}\left(\sqrt{-\hat{M}^{2} v^{2}}\right)
\end{aligned}
$$

For the discrete series things become a little bit more complicated, since, for example, $e^{i \sigma_{3} \varphi}$ is conjugate to $e^{-i \sigma_{3} \varphi}$ in $S L(2, \mathbb{C})$ but not in $S_{\beta}$. The 
integration in (3.52) may, however, be split up into two parts, in each of which Trace $D(\varrho(\alpha, p))$ may be considered as independent of $p$. Using the same techniques as above, one obtains for the Discrete Series

$$
\begin{aligned}
\text { Trace } U(\alpha, a)= & \frac{\left(\delta(\varphi)+(-1)^{2 x} \delta(\varphi-2 \pi)\right)}{4 \sinh ^{3} \frac{\eta}{2}} \times \\
& \times \frac{e^{-(2 x-1) \frac{\eta}{2}}}{2} \cdot 2 \pi J_{0}\left(\sqrt{-\hat{M}^{2} v^{2}}\right)+ \\
& +\frac{\pi \delta(\eta)(-1)^{\frac{1-\tau_{2}}{2}}}{8}\left(e^{i \tau_{2} \frac{\varphi}{2}(2 x-1)} H_{0}^{(1)}\left(\sqrt{-\hat{M}^{2} v_{-\sigma_{1}}^{2}}\right)+\right. \\
& \left.+e^{-i \tau_{2} \frac{\varphi}{2}(2 x-1)} H_{0}^{(1)}\left(\sqrt{-\bar{M}^{2} v_{+\sigma_{1}}^{2}}\right)\right) .
\end{aligned}
$$

If we denote that part of $(3.47)$ which is proportional to $\delta(\eta)$ by Trace ${ }^{I I I} U(\alpha, a)$, we have a relation similar to $(3.38)$ :

$\lim _{\hat{M} \rightarrow 0} \operatorname{Trace}{ }^{\mathrm{III}} U\left(\alpha, a ; \hat{M}^{2}, \varkappa, \tau_{2}\right)=\sum_{s^{\prime}=\varkappa}^{\infty}\left(\operatorname{Trace} U\left(\alpha, a ; P_{\mu} P^{\mu}=0, \tau_{2} s^{\prime},+\right)+\right.$

$$
+\operatorname{Trace} U\left(\alpha, a ; P_{\mu} P^{\mu}=0,-\tau_{2} s^{\prime},-1\right) \text {. }
$$

\section{The Representations with $P_{\mu} \equiv 0$}

The class IV representations are those, in which the translational subgroup of $\overline{\mathfrak{P}}$ is trivially represented, and any irreducible unitary representation of $S L(2, \mathbb{C})$ exactly defines such an irreducible representation of $\overline{\mathfrak{P}}$. For convenience, we only quote the Main series of representations $D(\alpha)$ of $S L(2, \mathbb{C})$ in the Hilbert space $\mathscr{H}=\{\psi(z, z)\},\|\psi\|^{2}$ $=\int \mid \psi(z, z)^{2} d u d v, z=u+i v$ :

with

$$
(D(\alpha) \psi)(z, \bar{z})=\mu(\alpha, z, \bar{z}) \psi\left(\frac{\alpha_{1}^{1} z+\alpha_{2}^{1}}{\alpha_{1}^{2} z+\alpha_{2}^{2}}, \frac{\overline{\alpha_{1}^{1} z+\alpha_{2}^{1}}}{\alpha_{2}^{1} z+\alpha_{2}^{2}}\right)
$$

$$
\begin{gathered}
\left.\mu(\alpha, z, \bar{z})=\left(\alpha_{1}^{2} z+\alpha_{2}^{2}\right)^{-i \sigma+j_{0}-1} \overline{\left(\alpha_{1}^{2} z+\alpha_{2}^{2}\right.}\right)^{-i \sigma+j_{0}-1} \\
j_{0}=0, \pm \frac{1}{2}, \pm 1, \ldots,-\infty<\sigma<+\infty .
\end{gathered}
$$

The different representations are parametrized by $\left(j_{0}, \sigma\right)$. Representations belonging to $\left(j_{0}, \sigma\right)$ and $\left(-j_{0},-\sigma\right)$ are equivalent. The characters have been calculated by NaImark et al. [3]:

Trace $U(\alpha, a)=\frac{1}{2\left|\lambda-\lambda^{-1}\right|^{2}}\left(|\lambda|^{-2\left(i \sigma+j_{0}\right)} \lambda^{2 j_{0}}+|\lambda|^{2\left(i \sigma+j_{0}\right)} \lambda^{-2 j_{0}}\right)$. 


\section{The Mathematical Framework}

\section{Definition and Properties of Induced Representations and Induced Characters}

All irreducible representations of $\overline{\mathfrak{P}}$ are induced representations in the sense of Frobenius and G. W. Mackey. For finite groups, Frobenius has given a formula to calculate the character of an induced representation from the character of the inducing representations. We shall show in the case of $\overline{\mathfrak{P}}$ how this procedure may be generalized for induced representations of locally compact groups with the help of the methods of distribution theory. So we gain a more rigorous framework for our preceding calculations.

The following is MackeY's definition of a representation $U(g)$ of a countable, locally compact group $G$ induced by a continuous unitary representation $D$ of a closed subgroup $F$ :

Let $D$ be a continuous unitary representation of $F, \mathscr{H}(D)$ the (separable) Hilbert-space of this representation. For $\Phi, \Psi \in \mathscr{H}(D)$ let $(\Phi, \Psi)$ be scalar product and let $\mu$ be the essentially unique quasiinvariant measure on the right cosetspace $G / F$. Let $\mathfrak{R}$ be the set of all maps $\Psi(g)$ from $G$ to $\mathscr{H}(D)$ such that

1. $(\Psi(g), \Phi)$ for each $\Phi \in \mathscr{H}(D)$ is a Borel-function in $g$,

2. $\Psi(f g)=D(f) \Psi(g), f \in F, g \in G$,

3. $\int_{G / F}(\Psi(g), \Psi(g)) d \mu(g)<\infty$.

Thus $\|\Psi(g)\|^{2}=(\Psi(g), \Psi(g))$ is a Borel-function on $G$ which is constant on the right cosets of $F$ in $G$. 3. defines a scalar product on $\mathfrak{R}$, by means of which $\mathfrak{R}$ becomes a separable Hilbert-space. The unitary representation $U(g)$ of $G$ in $\mathfrak{R}$ is then given by

where

$$
\left(U\left(g_{1}\right) \Psi\right)\left(g_{2}\right)=\Psi\left(g_{2} g_{1}\right) \sqrt{\varrho_{g_{1}}}\left(\overline{\left.g_{2}\right)} \quad g_{1}, g_{2} \in G\right.
$$

$$
\varrho_{g_{1}}\left(g_{2}\right)=\frac{d \mu\left(g_{2} g_{1}\right)}{d \mu\left(g_{2}\right)}
$$

is the Radon-Nikodym derivative of $\mu$.

Now we remark that the definition of the representations of $\overline{\mathfrak{P}}$ of type I, II and III as given in (3.2), (3.26) and (3.44) define induced representations in the sense of MAckey [6]. The subgroups $F_{i}$ $(i=\mathrm{I}, \mathrm{II}, \mathrm{III})$ are in the three cases the semi-direct products $(\mathrm{I})=S U(2) \cdot \mathfrak{T},(\mathrm{II}): S_{\gamma} \cdot \mathfrak{T},(\mathrm{III}): S_{\beta} \cdot \mathfrak{T}$. The inducing representations of these sub-groups $F_{i}$ are of the form

$$
D^{\prime}(s, a)=e^{i p_{i} a} \cdot D(s), \quad s \in S_{i} \cong F_{i} / / \mathfrak{T}=S U(2), S_{\gamma}, S_{\beta}
$$


with

$p_{\mathrm{I}}=(M, 0,0,0), p_{\mathrm{II}}=\left(\varepsilon_{2}, 0,0, \varepsilon_{2}\right), p_{\mathrm{III}}=(0,0,0, \hat{M}) \quad \Lambda_{s} p_{i}=p_{i}$

and the irreducible representations $D(s)$ of $S_{i}$ as discussed in $\left(3.4^{\prime}\right)$, (3.19), (3.20), (3.36) and (3.37). The Lorentz transformations $(\mathrm{I}): h(p)$, II $: t(p),(\mathrm{III}): r(p)$ defined in (3.3), (3.24) and (3.41) form systems of representatives of the $F_{i}$ right cosets $(\alpha, a)=(\varrho, a)(h(p), 0) ;(\varrho, a) \in F_{\mathrm{I}}$ etc. Therefore the coset spaces $\overline{\mathfrak{P}} / F_{i}$ are isomorphic to the unit momentum hyperboloids $\mathfrak{M}_{i}$; and the quasiinvariant measures $\mu$ are the invariant measures on these hyperboloids:

$$
\begin{aligned}
\text { (I) } \quad \mathfrak{M}_{\mathrm{I}} & =\left\{\hat{p} \mid \hat{p}^{2}=1, \varepsilon\left(p_{0}\right)=1\right\}, \quad d \mu(\hat{p})=\frac{d^{3} \hat{\mathbf{p}}}{2\left|\hat{p}_{0}\right|} \\
\text { (II) } \quad \mathfrak{M}_{\mathrm{II}} & =\left\{\hat{p} \mid \hat{p}^{2}=0, \varepsilon\left(p_{0}\right)=1\right\}, \quad d \mu(p)=\frac{d^{3} \hat{\mathbf{p}}}{2\left|\hat{p}_{0}\right|} \\
\text { (III) } \quad \mathfrak{M}_{\mathrm{III}} & =\left\{\hat{p} \mid \hat{p}^{2}=-1\right\}, d \mu(\hat{p})=\frac{|\mathbf{p}|}{2} d \hat{p}_{0} d \cos \vartheta d \varphi .
\end{aligned}
$$

Since $d \mu(p)$ is Lorentz invariant, the Radon-Nikodym derivative $\frac{d \mu\left(g_{2} g_{1}\right)}{d \mu\left(g_{2}\right)}$ is unity.

The functions $\Psi(g) \in \Omega$ which satisfy (4.1) are uniquely determined by their values on a system of representatives of the $F_{i}$ right cosets. Let us take, for example, representations $h(p)$ for type I as such representatives. Then we get from (4.2)

$$
\begin{aligned}
(U(\alpha, a) \Psi)(h(\hat{p})) & =D(\varrho(\alpha, \hat{p})) e^{i \varepsilon_{1} M \hat{p} a} \Psi\left(h\left(\Lambda_{\alpha}^{-1} \hat{p}\right)\right) \\
\varrho(\alpha, \hat{p}) & =h(\hat{p}) \alpha h^{-1}\left(\Lambda_{\alpha}^{-1} \hat{p}\right)
\end{aligned}
$$

which becomes identical with (3.2) by the correspondence $p \leftrightarrow M \hat{p}$, $(h(\hat{p})) \leftrightarrow \Psi\left(\frac{\varepsilon}{M} p\right)$. Similarly we see in the other cases II and III that the irreducible representations of $\overline{\mathfrak{P}}$ are induced representations.

We shape Frobenius' formula of the characters of induced representations of finite groups in a form which is a suitable analogue for the distribution theoretic discussion of the characters of $\overline{\mathfrak{P}}$. Let $G$ be a finite group with a sub-group $F$ and $\left\{g_{r} \mid r=1, \ldots,[G: F]\right\}$ be a system of representatives of the right cosets $G / F$. The group $G$ becomes a transformation group on the set $I=\{r\}$ of the indices of the cosets by the definition

$$
g \cdot g_{r}=g_{r_{g}} f \quad g \in G, f \in F
$$

With this notation the character of the representation of $G$ induced by a representation of $F$ with the Character $\chi_{F}(f)$ becomes $[7,13]$

$$
\chi_{G}(g)=\sum_{r \in I} \chi_{F}\left(g_{r} g g_{r}^{-1}\right) \delta_{r, r_{g}} .
$$

Since a representation of a group $G: g \rightarrow D(g)$ defines a representation 
of the group algebra $\mathbb{C} G: \sum_{g} \varphi(g) \cdot g \rightarrow D(\varphi)=\sum_{g} \varphi(g) D(g)$, we may consider the characters as linear functionals on the group algebra

$$
\chi_{G}(\varphi)=\operatorname{Trace}(D(\varphi))=\sum_{g} \varphi(g) \chi_{G}(g) \text {. }
$$

The relation between the characters $\chi_{G}(\varphi)$ and $\chi_{H^{\prime}}(\psi)$ of the group algebras $\mathbb{C} G=\{\varphi(g)\}$ and $\mathbb{C} F=\{\psi(f)\}$, which corresponds to Eq. (4.7) may be expressed with the help of the following mapping of $G \times \mathrm{I} \times \mathrm{I}$ into $\mathbb{C} F$ :

It becomes

$$
\psi_{r r^{\prime}}(f)=\varphi\left(g_{r}^{-1} f g_{r^{\prime}}\right)
$$

$$
\chi_{G}(\varphi)=\sum_{r \in I} \chi_{F^{\prime}}\left(\psi_{r r}\right)
$$

This is the form in which Frobenius' formula may be generalized to the induced representations of $\overline{\mathfrak{P}}$. We must study suitable spaces of test functions on $\overline{\mathfrak{P}}$ (section IV, 2) and on the inducing groups (section IV, 3). The discussion of the mapping (4.9) helps to demonstrate that we may define the characters of $\overline{\mathfrak{P}}$ as distributions of the type (4.10).

\section{The Space A of Test Functions on $\overline{\mathfrak{P}}$}

The Poincaré group $\overline{\mathfrak{P}}$ is an analytic Lie group, and its analytic structure is given by the direct product of the analytic structures of $S L(2, \mathbb{C})$ and $\mathfrak{T}$. For our purpose it is useful to construct a space of test functions $A$ which has the properties of L. ScHwarTz' space [14] $\mathscr{S}$ with respect to $\mathbb{S}$ and of his space $\mathscr{D}$ with respect to $S L(2, \mathbb{C})$. We use a finite atlas $\mathfrak{A}$ in $\overline{\mathfrak{P}}$ given by the canonical coordinate system in $\mathfrak{T}$ and a finite atlas $\mathfrak{A}(S L(2, \mathbb{C}))^{2}$. Now let $A_{K}$ be the set of all complex valued function $f(\alpha, a)$ on $\overline{\mathfrak{P}}$ which are infinitely differentiable and which have a support contained in $K \times \mathfrak{T}$, where $K$ is any compact set in $S L(2, \mathbb{C})$. Furthermore, we suppose that $f(\alpha, a)$ and all its partial derivatives decrease faster than any inverse power of $|a|^{2}=a_{0}^{2}+a_{1}^{2}+a_{2}^{2}+a_{3}^{2}$ for $|a|^{2} \rightarrow \infty$ uniformly in $\alpha . A_{K}$ is not empty; we make $A_{K}$ a locally convex topological vector space by defining the following basis of neighbourhoods $V$ of the zero in $A_{K}$ :

$$
\begin{aligned}
V(m, n, q ; \varepsilon, \mathfrak{A}) & \\
& =\left\{f(\alpha, a) \in A_{K} \sup _{K \times \mathfrak{I}}\left(\left(1+|a|^{2}\right)^{m}\left|\frac{\partial^{n}}{\partial \alpha^{n}} \frac{\partial^{\alpha}}{\partial a^{q}} f(\alpha, a)\right|\right)<\varepsilon\right\}
\end{aligned}
$$

$m, n, q$ are non-negative integers, $\frac{\partial^{n}}{\partial \alpha^{n}} \frac{\partial^{q}}{\partial a^{q}}$ denote any partial derivative of order $(n+q)$ in any coordinate system contained in $\mathfrak{A} . A_{K}$ is a Montel space, the topology is independent of the special choice of $\mathfrak{A}$. We define $A=\lim \operatorname{ind}_{K} A_{K}$. The space $A$ is a locally convex topological vector space in which $A_{K}$ is a closed linear subspace. By definition, a convex

${ }^{2}$ There is an atlas with only two coordinate systems. 
set $U$ in $A$ is a neighbourhood of zero if and only if $U \cap A_{K}$ is a (convex) neighbourhood of zero in $A_{K}$. Therefore, a linear mapping $F$ from $A$ into a locally convex topological vector space is continuous if and only if the restriction $F_{K}$ of $F$ to $A_{K}$ is continuous for each $K$. Then $F$ maps bounded sets into bounded sets. A set $M$ in $A$ is bounded if and only if all functions $f \in M$ belong to some $A_{K}$ and the supremum with respect to $f, \alpha$ and $a$ of the partial derivatives of any order multiplied by $\left(1+|a|^{2}\right)^{n}-$ with $n$ arbitrary - is always bounded.

The space $A$ may be regarded as a dense *-subalgebra of the group algebra $L^{1}\left(\overline{\mathfrak{P}}, d \mu(\alpha) d^{4} a\right)$. The convolution is defined in the usual manner:

$$
\begin{aligned}
\left(f \circ f^{\prime}\right)(\alpha, a) & =\int_{\frac{\mathcal{P}}{\mathcal{F}}} f\left((\alpha, a)(\alpha, a)^{-1}\right) f\left(\alpha^{\prime}, a^{\prime}\right) d \mu\left(\alpha^{\prime}\right) d^{4} \alpha^{\prime} \\
f^{*}(\alpha, a) & =\overline{f\left((\alpha, a)^{-1}\right)} .
\end{aligned}
$$

Since the convolution maps $A_{K} \times A_{K^{\prime}}$ into $A_{K K^{\prime}}$ and the involution maps $A_{K}$ into $A_{K^{-1}}$ it can be shown that both operations are continuous with respect to the topology in $A$.

In $A$ there exists an approximation of the unit $\left\{e_{n}\right\}$ [14], such that $e_{n} \in A$ and

$$
\int_{\mathfrak{P}} e_{n}(\alpha, a) d \mu(\alpha) d^{4} a=1 ; \lim _{n \rightarrow \infty} e_{n} \circ f=\lim _{n \rightarrow \infty} f \circ e_{n}=f
$$

for each $f \in A$. We may assume that the $e_{n}$ have compact supports $K_{n}$ in $\overline{\mathfrak{P}}$ which satisfy $\bigcap_{n} K_{n}=(1,0)$.

The Fourier transform with respect to the translations $\mathfrak{F}$ :

$$
f(\alpha, p)=\int_{\mathfrak{T}} f(\alpha, a) e^{i p a} d^{4} a
$$

is a linear homeomorphism of $A$ onto itself [14].

Now we define distributions as continuous linear functionals on $A$. The set of all distributions is denoted by $A^{\prime}$. We call $\chi \in A^{\prime}$ real, if $\chi\left(f^{*}\right)=\overline{\chi(f)}$, positive if $\chi\left(f \circ f^{*}\right)>0$ and central, if $\chi\left(f \circ f^{\prime}\right)=\chi\left(f^{\prime} \circ f\right)$ for all $f, f^{\prime} \in A$. The real distributions form a real vector space, the positive distributions form a positive cone and the central distributions form a complex vector space in $A^{\prime}$. We expect the primitive characters of $\bar{P}$ to be real, positive, central distributions on $A$. We remark that a real, positive central distribution $\chi$ is uniquely determined by its values on the positive elements $\chi\left(f \circ f^{*}\right)$. In order to see this, we calculate $\chi\left(f \circ f^{\prime}\right)$ by polarization and use then the approximation of the unit to get $\chi(f)=\lim _{n \rightarrow \infty}\left(f \circ e_{n}\right)$.

Let $(\alpha, a) \rightarrow U(\alpha, \alpha)$ be any continuous homomorphism of $\overline{\mathfrak{P}}$ into the group of all unitary operators of a Hilbert space $\mathscr{H}$, then

$$
T_{f}=\int_{\mathfrak{P}} f(\alpha, a) U(\alpha, a) d \mu(\alpha) d^{4} a
$$


defines a continuous $*$-homomorphism of $A$ into the $*$-algebra of the bounded operators in $\mathscr{H}$ :

$$
T_{j^{*}}=T_{f}^{+} ; \quad T_{f \circ f^{\prime}}=T_{f} T_{f^{\prime}} .
$$

We shall consider the properties of $T_{f}$ in the following.

\section{The Test Function Spaces on $S U(2), S_{\beta}$ and $S_{\gamma}$}

We are dealing with induced representations, and we need therefore the locally convex topological vector spaces of the test functions on the little groups. Let $S$ be any of the unimodular groups $S U(2), S_{\beta}$ or $S_{\gamma}$. Consider the linear space $\mathscr{D}$ consisting of all complex valued infinitely differentiable functions $\hat{f}$ on $S$ with compact support. We furnish $\mathscr{D}$ with the topology given by L. Schwartz [14]. Similar as above, we use a finite atlas. The topology, however, is independent of the special choice of that atlas.

Just as above, we may define a convolution and involution in $\mathscr{D}$ :

$$
\begin{aligned}
\left(\hat{f} \times \hat{f}^{\prime}\right)(s) & =\int_{S} \hat{f}\left(s s^{\prime-1}\right) \hat{f}^{\prime}\left(s^{\prime}\right) d \mu\left(s^{\prime}\right) \\
\hat{f}^{*}(s) & =\overline{f\left(s^{-1}\right)}
\end{aligned}
$$

where $d \mu(s)$ is the Haar measure on $S$. For any continuous homomorphism of $S$ into the group of all unitary operators of a Hilbert space $H: s \rightarrow U(s)$, we define by

$$
\hat{T}_{\hat{f}}=\int_{S} U(s) \hat{f}(s) d \mu(s)
$$

a continuous $*$-homomorphism of $\mathscr{D}$ into the $*$-algebra of all bounded operators in $H$ :

$$
\hat{T}_{\hat{f}}=\hat{T}_{\hat{f}^{+}} ; \quad \hat{T}_{\hat{f} * \hat{f}^{\prime}}=\hat{T}_{\hat{f}} \hat{T}_{\hat{f}^{\prime}} .
$$

Now we consider the characters of $S$ as distributions. For the irreducible representations of $S U(2)$ and for the representations of the Main and Discrete series of $S_{\beta}$ it is known that there are continuous linear, real, positive and central distributions $\hat{\chi}$ on $\mathscr{D}$, such that $\hat{\chi}$ is the trace for the positive elements of $\mathscr{D}[12 \mathrm{~b}]$ :

$$
\hat{\chi}\left(\hat{f} \times \hat{f}^{*}\right)=\operatorname{Trace} \hat{T}_{\hat{f} \times \hat{f}^{*}}=\operatorname{Trace}\left(T_{\hat{f}} T_{\hat{f}}^{+}\right) .
$$

In the remaining case of $S=S_{\gamma}$, the one dimensional representations (3.19) define distributions on $\mathscr{D}$

$$
\hat{\chi}(\hat{f})=\int_{0}^{4 \pi} e^{i s \varphi} \hat{f}(\varphi, u) d \varphi d u
$$

with all the desired properties. The Equation (4.19) reads for the faithful representations of $S_{\gamma},(3.20)$ :

$$
\begin{aligned}
\left(\hat{T}_{\hat{f}} \Psi\right)(v)= & \int_{0}^{2 \pi} K\left(\hat{f} \mid v, v^{\prime}\right) \Psi\left(v^{\prime}\right) d v^{\prime} \\
K\left(\hat{f} \mid v, v^{\prime}\right)= & \int \exp \left[i Q \cdot \operatorname{Re}\left(e^{i \gamma} u\right)\right] \cdot \hat{f}\left(v^{\prime}-v, u\right) d u+ \\
& +(-1)^{\tau} \int \exp \left[i Q \cdot \operatorname{Re}\left(e^{i v} u\right) \hat{f}\left(v^{\prime}-v+2 \pi, u\right) d u .\right.
\end{aligned}
$$


Defining

$$
\hat{\chi}(\hat{f})=\text { Trace } \hat{T}_{\hat{f}}=\frac{1}{2 \pi} \int_{0}^{2 \pi} K(f \mid v, v) d v
$$

we obtain our old distribution (3.21)

$\hat{\chi}(f)=\int\left\{\delta(\varphi)+(-1)^{\tau} \delta(\varphi-2 \pi)\right\} 2 \pi J_{0}(Q|u|) \hat{f}(\varphi, u) d \varphi d u_{1} d u_{2}$.

The continuity of $\hat{\chi}$ follows from the estimate we get from (4.21) and (4.24)

$$
|\hat{\chi}(f)| \leqq 4 \pi \sup _{\gamma(\varphi, u) \in \hat{K}}|\hat{f}(\varphi, u)| \int_{\gamma(\varphi, u) \in \hat{K}} d u_{1} d u_{2}
$$

where $\hat{K}$ is the support of $\hat{f}$.

Thus for all little groups $S_{i}$ we have found real, positive central distributions on the corresponding spaces $\mathscr{D}$, which on the positive elements agree with traces $\hat{\chi}\left(\hat{f} \times \hat{f}^{*}\right)=\operatorname{Trace}\left(T_{\hat{f}} T_{\hat{f}}^{+}\right)$. We denote in the following these distributions as generalized traces

$$
\hat{\chi}(\hat{f})=\overline{\text { Trace }} \hat{T}_{\hat{f}} .
$$

\section{The Characters of $\overline{\mathfrak{P}}$ for Representations with $P_{\mu} \neq 0$}

We start the discussion of this section by rewriting Formula (4.15)

$$
\begin{aligned}
T_{f} \Psi(p) & =\int_{\mathfrak{P}} f(\alpha, a) D^{\prime}\left(l_{i}(p)(\alpha, a) l_{i}^{-1}\left(\Lambda_{\alpha}^{-1} p\right)\right) \Psi\left(\Lambda_{\alpha}^{-1} p\right) d \mu(g) \\
l_{i}(p) & =(h(p), 0),(t(p), 0),(r(p), 0) \text { for } i=\text { I, II, III } .
\end{aligned}
$$

with the help of the factorization of the measure $d \mu(g)$ of $\overline{\mathfrak{P}}$ in the measure of the inducing subgroup $F_{i}: d \mu(s) d a$ and the invariant measure $d \mu(p)$ on the right coset space $\mathfrak{P l}_{i}=\overline{\mathfrak{P}} / F_{i}$, i.e. $d \mu(g)=d \mu(s) \times$ $\times d a d \mu(p)$. We get

with the kernels

$$
T_{f} \Psi(p)=\int_{\mathfrak{M}_{i}} K\left(f \mid p, p^{\prime}\right) \Psi\left(p^{\prime}\right) d \mu\left(p^{\prime}\right)
$$

$$
\begin{aligned}
\text { (I) } K\left(f \mid p, p^{\prime}\right) & =\int_{S U(2) \times \mathfrak{T}} f\left(h^{-1}(p) u h\left(p^{\prime}\right) ; h^{-1}(p) a\right) e^{i \varepsilon_{1} M a_{0}} D(u) d \mu(u) d a \\
& =\int_{S U(2)} \tilde{f}\left(h^{-1}(p) u h\left(p^{\prime}\right) ; \varepsilon_{1} M p\right) D(u) \cdot d \mu(u) \\
\text { (II) } K\left(f \mid p, p^{\prime}\right) & =\int_{S_{\gamma}} \tilde{f}\left(t^{-1}(p) \gamma t\left(p^{\prime}\right) ; \varepsilon_{2} p\right) D(\gamma) \cdot d \mu(\gamma) \\
\text { (III) } K\left(f \mid p, p^{\prime}\right) & =\int_{S_{\beta}} \tilde{f}\left(\tilde{r}^{-1}(p) \beta r\left(p^{\prime}\right) ; \hat{M} p\right) D(\beta) d \mu(\beta) .
\end{aligned}
$$

The transformation $h(p)$ is defined on $\mathfrak{W}_{\mathrm{I}}$ and depends there analytically on $p ; t(p)$ is defined in the region $p_{+} \neq 0, p_{-} \neq 0$ of $\mathfrak{R}_{\mathrm{II}}$ and is analytic there; similarly $r(p)$ is defined for $p_{3} \neq 0$ on $\mathfrak{W}_{\mathrm{III}}$ and is analytic there. 
For $p, p^{\prime}$ in these regions of definition $\mathfrak{V}_{i}$ of $h(p), t(p)$ and $t(p)$, the integration with respect to $d \mu(u)$ is performed over a compact set. Therefore, for fixed $\left(p, p^{\prime}\right), f\left(h^{-1}(p) u h\left(p^{\prime}\right) ; \varepsilon_{1} M p\right)$ is a $C^{\infty}$-function on $S U(2) \cap$ $\cap h(p) K h^{-1}\left(p^{\prime}\right)$, whenever $f \in A_{K}$. We have a similar situation in the other cases. Therefore the kernels defined in $(4.27 \mathrm{a}, \mathrm{b}, \mathrm{c})$ exist except on a set of measure zero and define bounded linear operators in the corresponding representation spaces $\mathscr{H}(D)$. We have to prove several properties of these operators.

Lemma 1. $K\left(f \mid p, p^{\prime}\right)$ is continuous in $\left(p, p^{\prime}\right)$ whenever defined.

Proof. Let $B$ and $B^{\prime}$ be any compact neighbourhoods of $p$ and $p^{\prime}$ in $\mathfrak{V}_{i}$. Then for each $\left(\bar{p}, \bar{p}^{\prime}\right)$ in $B \times B^{\prime}$, the functions in the integrands of (4.27) depend continuously on $\left(\bar{p}, \bar{p}^{\prime}\right)$ and have support in the compact sets $h(B) K h^{-1}\left(B^{\prime}\right) \cap S U(2), t(B) K t^{-1}\left(B^{\prime}\right) \cap S_{\gamma}, r(B) K r^{-1}\left(B^{\prime}\right) \cap S_{\beta}$ respectively for all $f \in A_{K}$. Therefore the integration in $(4.27 \mathrm{a}, \mathrm{b}, \mathrm{c})$ may be extended over these sets only. The lemma follows now from a wellknown theorem in analysis [15] for all $\left(\bar{p}, \bar{p}^{\prime}\right)$ in the interior of $B \times B^{\prime}$.

\section{Lemma 2.}

$$
\begin{aligned}
K\left(f^{*} \mid p, p^{\prime}\right) & =K\left(f \mid p^{\prime}, p\right)^{+} \\
K\left(f \circ f^{\prime} \mid p, p^{\prime}\right) & =\int K\left(f \mid p, p^{\prime \prime}\right) K\left(f^{\prime} \mid p^{\prime \prime}, p^{\prime}\right) d \mu\left(p^{\prime \prime}\right) .
\end{aligned}
$$

Proof. Eq. (4.28a) follows immediately from the definitions (4.12) and (4.27). For the proof of the second statement we shall show certain falloff properties which justify the following calculation

$K\left(f \circ f^{\prime} \mid p, p^{\prime}\right)$

$=\int f(\alpha, a) f^{\prime}\left((\alpha, a)^{-1}\left(l^{-1}(p) s l\left(p^{\prime}\right), \Lambda_{l(p)}^{-1} a^{\prime}\right)\right) e^{i p_{i} a^{\prime}} D(s) d \mu(\alpha) d a d \mu(s) d a^{\prime}$ we substitute $l(p) \alpha=s^{\prime} l\left(p^{\prime \prime}\right), \Lambda_{s^{\prime}}^{-1} a^{\prime}-\Lambda_{l^{-1},(p) s^{\prime}}^{-1} a=b, \Lambda_{l(p)} a=b^{\prime}$, we use $s^{\prime} p_{i}=p_{i}$ and we get

$$
\begin{aligned}
& K\left(f \circ f^{\prime} \mid p, p^{\prime}\right) \\
& \quad=\int f\left(l^{-1}(p) s^{\prime} l\left(p^{\prime \prime}\right), \Lambda_{l(p)}^{-1} b^{\prime}\right) f^{\prime}\left(l^{-1}\left(p^{\prime \prime}\right) s^{\prime-1} s l\left(p^{\prime}\right), \Lambda_{l\left(p^{\prime \prime}\right)}^{-1} b\right) e^{i p_{i}\left(b+b^{\prime}\right)} \times \\
& \quad \times D\left(s^{\prime}\right) D\left(s^{\prime-1} s\right) d \mu\left(s^{\prime}\right) d \mu\left(p^{\prime \prime}\right) d \mu(s) d b d b^{\prime} .
\end{aligned}
$$

According to definition (4.27) this expression agrees with (4.28b) if we interchange the order of integration.

Now we discuss the fall-off properties which allow this manipulations for the different cases.

(I) The kernels $K\left(f \mid p, p^{\prime \prime}\right)$ and $K\left(f^{\prime} \mid p^{\prime \prime}, p^{\prime}\right)$ of the form (4.27a) are zero for fixed $\left(p, p^{\prime}\right)$ and sufficiently large $p^{\prime \prime}$, because $h\left(p^{\prime \prime}\right) \rightarrow \infty$ as $p^{\prime \prime} \rightarrow \infty$ and $S U(2)$ is compact. Therefore the integration in (4.28 b) may be performed.

(III) In order to discuss $K\left(f \mid p, p^{\prime \prime}\right)$ and $K\left(f^{\prime} \mid p^{\prime \prime}, p^{\prime}\right)$ of the form $(4.27 \mathrm{c})$ we make use of the fact that the set $\underset{p, p^{\prime} \in \mathfrak{V}_{3}}{\bigcup} r(p) K r^{-1}\left(p^{\prime \prime}\right) \cap S_{\beta}$ is 
bounded, as we have shown in Appendix $\mathrm{C}$. There is thus a $C(K)$ such that

$$
\mu_{S_{\beta}}\left(r(p) K r^{-1}\left(p^{\prime \prime}\right) \cap S_{\beta}\right)<C(K)
$$

and therefore the norm of $K\left(f \mid p, p^{\prime \prime}\right)$ as an operator in $\mathscr{H}(D)$ satisfies

$$
\left\|K\left(f \mid p, p^{\prime \prime}\right)\right\|<C(K) \sup _{\beta \in S_{\beta}}\left|f\left(r^{-1}(p) \beta r\left(p^{\prime \prime}\right), \hat{M} p\right)\right| .
$$

As a consequence $K\left(f \mid p, p^{\prime \prime}\right)$ goes to zero faster than any inverse power in $\left(1+|p|^{2}\right)$ as $p \rightarrow \infty$ uniformly in $p^{\prime \prime}$. Since we have $K\left(f \mid p, p^{\prime \prime}\right)$ $=K\left(f^{*} \mid p^{\prime \prime}, p\right)^{\dagger}$ and $\left\|K\left(f \mid p, p^{\prime \prime}\right)\right\|=\left\|K\left(f^{*} \mid p^{\prime \prime}, p\right)\right\|$ the properties of $\left\|K\left(f \mid p, p^{\prime \prime}\right)\right\|$ are symmetric in $p, p^{\prime \prime}$. From this and from corresponding properties of $K\left(f^{\prime} \mid p^{\prime \prime}, p\right)$ we conclude that the integral in (4.28b) exists also in this case.

(II) The case $P^{2}=0$ is more complicated, because $t(p) \rightarrow \infty$ as $p \rightarrow 0$. It is shown in Appendix C2 that there is a $C^{\prime}(K)$ such that

and therefore

$$
\begin{gathered}
\int_{\mathfrak{W}} d u_{1} d u_{2} \leqq \frac{C^{\prime}(K)}{|\mathbf{p}|\left|\mathbf{p}^{\prime}\right|} \\
\mathfrak{W}=\left\{u_{1}, u_{2} \mid \gamma(\varphi, u) \in t(p) K t^{-1}\left(p^{\prime}\right) \cap S_{\gamma}\right\}
\end{gathered}
$$

$$
\mu_{s_{\gamma}}\left(t(p) K t^{-1}\left(p^{\prime}\right) \cap S_{\gamma}\right) \leqq 4 \pi \cdot \frac{C^{\prime}(K)}{|\mathbf{p}|\left|\mathbf{p}^{\prime}\right|}
$$

Thus $K\left(f \mid p, p^{\prime \prime}\right)$ goes to zero faster than any inverse power in $\left(1+|p|^{2}\right)$ as $p \rightarrow \infty, p^{\prime \prime}$ fixed. Using the symmetry argument from above, we see that $K\left(f \mid p, p^{\prime \prime}\right)$ decreases fast also for fixed $p^{\prime}$ and $p^{\prime \prime} \rightarrow \infty$. In Appendix C2 it is further shown that $K\left(f \mid p, p^{\prime}\right)$ vanishes for $p^{\prime \prime} \rightarrow 0$ and fixed $p$ as well as for $p \rightarrow 0$ and fixed $p^{\prime \prime}$. Therefore integration (4.28b) may be also performed in this case also.

This finishes the proof of lemma 2 .

In order to understand the structure of the induced characters, we have to study the relation between the test functions on $\overline{\mathfrak{P}}$ and the test functions on the subgroups $S_{i}$. For this we consider the following mappings of $A \times \mathfrak{V}_{i} \times \mathfrak{V}_{i} \rightarrow \mathscr{D}$ :

$$
\left(f, p, p^{\prime}\right) \rightarrow \hat{f}_{p, p^{\prime}}=\left\{\begin{array}{l}
f\left(h^{-1}(p) \operatorname{sh}\left(p^{\prime}\right) ; \varepsilon_{1} M p\right) \\
f\left(t^{-1}(p) \gamma t\left(p^{\prime}\right) ; \varepsilon_{2} p\right) \\
f\left(r^{-1}(p) \beta r\left(p^{\prime}\right) ; \hat{M} p\right)
\end{array}\right.
$$

for the representations of the different types I, II, III. The test function spaces $A$ and $\mathscr{D}$ are defined in the sections 4.1 and 4.2 respectively.

Lemma 3. $\left(f, p, p^{\prime}\right) \rightarrow \hat{f}_{p, p^{\prime}}$ is continuous in $\left(f, p, p^{\prime}\right)$.

Proof. We note first that $\hat{f}_{p, p^{\prime}}$ is linear in $f$ for all $\left(p, p^{\prime}\right)$. Furthermore, for fixed $f, f_{p p^{\prime}}$ is obviously continuous in $p, p^{\prime}$. It is therefore sufficient to prove that for given $\left(p, p^{\prime}\right)$ and a given convex neighbourhood $\hat{U}$ of zero in $\mathscr{D}$ there is a convex neighbourhood $U$ of zero in $A$ and a neigh- 
bourhood $B \times B^{\prime}$ of $\left(p, p^{\prime}\right)$ such that $\hat{f}_{\bar{p}, \bar{p}^{\prime}} \in \hat{U}$ whenever $f \in U$ and $\left(\bar{p}, \bar{p}^{\prime}\right) \in B \times B^{\prime}$. We choose $B$ and $B^{\prime}$ to be compact neighbourhoods of $p$ and $p^{\prime}$ and we will show that $f \rightarrow \hat{f}_{\bar{p}}, \bar{p}^{\prime}$ is equicontinuous for $\left(\bar{p}, \bar{p}^{\prime}\right) \in$ $\in B \times B^{\prime}$. This will prove the lemma.

Since for fixed $\left(p, p^{\prime}\right)$ the map is linear in $f$, we may consider its restriction to $A_{K}$. We denote by $\mathscr{D}_{\hat{K}}$ the closed subspace of $\mathscr{D}$ which consists of all functions of $\mathscr{D}$ with support in the compact set $\hat{K} \subset S_{i}$.

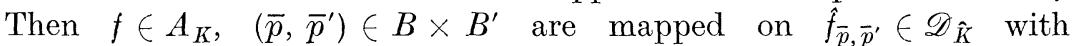
$\hat{K}=h(B) K h^{-1}\left(B^{\prime}\right) \cap S U(2), r(B) K r^{-1}\left(B^{\prime}\right) \cap S_{\beta}, t(B) K t^{-1}\left(B^{\prime}\right) \cap S_{\gamma}$ respectively. Now let $\hat{U}$ be a convex neighbourhood of zero in $\mathscr{D}$; there is a convex neighbourhood of zero $\hat{U}_{\hat{K}} \subset \hat{U} \cap \mathscr{D}_{\hat{K}}$ of the form

$$
\hat{U}_{\hat{K}}=\left\{\hat{f} \in \mathscr{D}_{\hat{K}} \mid \sup _{0 \leqq n \leqq m, s \in S}\left(\left|\frac{\partial^{n}}{\partial s^{n}} \hat{f}(s)\right|\right)<\varepsilon\right\}
$$

for some $m$ and $\varepsilon$. Here $\frac{\partial^{n}}{\partial s^{n}}$ denotes any partial derivative of order $n$ with respect to any coordinate system of a fixed finite atlas of $S$. We choose now a coordinate system $\alpha_{j}, j=1,2, \ldots, 6$, of $\mathfrak{A}(S L(2, \mathbb{C}))$ and a constant $C$ which is an upper bound to all kinds of products of partial derivatives $\frac{\partial^{n}}{\partial s^{n}} \alpha_{j}\left(l^{-1}(\bar{p}) s l\left(\bar{p}^{\prime}\right)\right)$ with respect to the parameters of $S$ :

$$
\sup _{\mathscr{J}}\left(\frac{\partial n_{j_{1}}}{\partial s^{n_{j_{1}}}} \alpha_{j_{1}}\left(l^{-1}(\bar{p}) s l\left(\bar{p}^{\prime}\right)\right) \cdot \ldots \cdot \frac{\partial^{n} j_{K}}{\partial s^{n_{j_{k}}}} \alpha_{j_{K}}\left(l^{-1}(\bar{p}) s l\left(\bar{p}^{\prime}\right)\right)\right)<C .
$$

Here the index $\mathscr{J}$ stands for the conditions: $\left(\bar{p}, \bar{p}^{\prime}\right) \in B \times B^{\prime}$,

$$
s \in \hat{K}, 1 \leqq j_{1} \leqq \cdots \leqq j_{k}=6,0<\sum_{i=1}^{k} n_{j i} \leqq m .
$$

Then for the neighbourhood $U_{K} \subset A_{K}$ :

$$
U_{K}=\left\{f \in A_{K}\left|\sup _{\substack{(\alpha, p) \in K \cdot \mathfrak{T} \\ 0 \leqq n \leqq m}}\left(\mid \frac{\partial^{n}}{\partial \alpha^{n}} f(\alpha, p)\right)\right|<\frac{\varepsilon C^{-1}}{6^{m} m !}\right\}
$$

we can easily get the statement, that $\hat{f}_{\bar{p}, \bar{p}^{\prime}} \in \hat{U}_{\hat{K}}$ for all $f \in U_{K}$. This completes the proof of lemma 3 .

After all these preparations we can determine the primitive characters of the Poincaré group as distributions over the test-function space $A$. With help of the definitions (4.18), (4.27) and (4.32) we may write

$$
K\left(f \mid p, p^{\prime}\right)=\hat{T}_{\hat{p_{p, p^{\prime}}}}
$$

and we get for the generalized trace in the sense of $(4.26)$ of $K\left(f \mid p, p^{\prime}\right)$ as operator in $\mathscr{H}(D)$

$$
\overline{\operatorname{Trace}} K\left(f \mid p, p^{\prime}\right)=\overline{\text { Trace }} \hat{T}_{f_{p, p^{\prime}}}=\hat{\chi}\left(\hat{f}_{p, p^{\prime}}\right) .
$$

This function is continuous in $f, p, p^{\prime}$ because of lemma 3 and our state- 
ment (4.26). We shall show below that

$$
\int_{\mathfrak{M}} \overline{\operatorname{Trace}} K(f \mid p, p) d \mu(p)=\int_{\mathfrak{Z}} \hat{\chi}\left(\hat{f}_{p}, p\right) d \mu(p)=\chi(f)
$$

is well defined and determines a distribution on $A$. The distribution $\chi(f)$ is the generalized trace of the representation of the group algebra $A$ of $\overline{\mathfrak{P}}$ related to the irreducible representations of type I, II, III, because for positive elements we calculate with help of Eq. (4.27) and lemma 2

$$
\chi\left(f \circ f^{*}\right)=\int_{\mathfrak{M} \times \mathfrak{M}} \operatorname{Trace}\left(K\left(f \mid p, p^{\prime}\right) K\left(f \mid p, p^{\prime}\right)^{+}\right) d \mu(p) \cdot d \mu\left(p^{\prime}\right) .
$$

The expression $(4,35)$ of $\chi(f)$ is very similar to the form $(4,10)$ of the induced character of finite groups. It represents a distribution theoretic generalization of this formula.

We shall give this result as a theorem.

Theorem. Let $U(g)$ be a unitary irreducible representation of $\overline{\mathfrak{P}}$ induced by a unitary representation of one of the subgroups $S_{i} \cdot \mathfrak{T}: S U(2) \cdot \mathfrak{T}$, $S L(2, \mathbb{R}) \cdot \mathfrak{T}, S_{\gamma} \cdot \mathfrak{T}$, of the form $D(s) e^{i p_{i} a}$ with the character $\hat{\chi}_{i}(s)$ of $D(s)$. The representation of the group algebra $A$ of $\overline{\mathfrak{P}}$ defined by $U(g)$ is $T_{f}=\int U(g) f(g) d \mu(g) ; f \rightarrow f_{p, p}$, denotes the family of mappings of $A$ in $\mathscr{D}$ as defined in (4.32). Then

$$
\chi_{i(f)}=\int_{\mathfrak{S}_{i}} \hat{\chi}\left(\hat{f}_{p p}\right) d \mu(p) \quad i=\mathrm{I}, \mathrm{II}, \mathrm{III}
$$

defines distributions on $A$ in the cases $i=I, I I I$ and continuous functionals on the closed *-ideal $A_{0}=\{f \in A \mid \tilde{f}(\alpha, 0)=0\}$ in the case $i=I I$. This distribution is the trace on the positive elements of $A$ resp. $A_{0}$

$$
\chi_{i}\left(f \circ f^{*}\right)=\operatorname{Trace}\left(T_{f} T_{f}^{\dagger}\right) \text {. }
$$

Proof. We have to show for the different cases that the integral in (4.37) defines a distribution on $A$.

(I) In Appendix C3 we have shown for $p \in \mathfrak{V}_{\mathrm{I}}$ and for fixed $f$ that $f_{p p}$ is a bounded family in $\mathscr{D}(S U(2))$ which goes in $\mathscr{D}(S U(2))$ strongly to zero as $p \rightarrow \infty$. This means, for a given $n$ and a given neighbourhood $\hat{U}$ of zero in $\mathscr{D}(S U(2))$, that there is an $N$ such that

for all $|p|^{2}>N$.

$$
\left(1+|p|^{2}\right)^{n} \hat{f}_{p p} \in \hat{U}
$$

(III) In the case $p \in \mathfrak{M}_{\mathrm{III}}$, we have seen that $\bigcup_{p, p^{\prime} \in \mathfrak{V}_{\mathrm{III}}} r(p) K r^{-1}\left(p^{\prime}\right) \cap S_{\beta}$ is bounded. Therefore we could show in Appendix C3 that for fixed $f$ the family of functions $\hat{f}_{p p}$ is bounded in $\mathscr{D}\left(S_{\beta}\right)$ and goes strongly to zero in $\mathscr{D}\left(S_{\beta}\right)$ as $|p| \rightarrow \infty$.

In both these cases it follows that $\hat{\chi}\left(\hat{f}_{p p}\right)$ is bounded and strongly decreasing and also continuous (Lemma 3 ) at almost all points. Hence 
we may integrate $\hat{\chi}\left(\hat{f}_{p p}\right)$ over $p$ and get thus a functional on $A$. The continuous dependence of the integral on $f$ follows from an argument similar to one used in the proof of lemma 1.

(II) The case $p \in \mathfrak{M}_{\text {II }}$ makes some trouble because it is possible that $\hat{f}_{p p}$ is not a bounded family for fixed $f$. Let $N_{\varepsilon}=\{p|| \mathbf{p} \mid>\varepsilon\}$, then the family $\left\{\hat{f}_{p p}\right\}_{p \in N_{\varepsilon} \cap \mathfrak{V}_{\mathrm{II}}}$ is bounded and $f_{p p}$ goes to zero strongly in $\mathscr{D}\left(S_{\gamma}\right)$ as $|p| \rightarrow \infty$. This is proved in Appendix C3. The support of $\hat{f}_{p p}$ may become arbitrary large as $|p| \rightarrow 0$, but, however, its measure does not diverge faster than $|\mathbf{p}|^{-2}$. But with the equations (4.25), (4.27 b), (4.31 a) (4.33) and $d \mu(p)=\frac{|\mathbf{p}|}{2} d|\mathbf{p}| d \Omega_{\mathbf{p}}$ in mind, we see that the limit

$$
\lim _{\varepsilon \rightarrow 0} \int_{N_{\varepsilon} \cap \mathcal{V}_{I I}} \hat{\chi}\left(\hat{f}_{p p}\right) d \mu(p)
$$

exists for all $f$ with $f(\alpha, 0)=0$. On this subspace the linear functional so obtained is continuous.

This theorem gives a justification of our formal calculations of chapter III. There we calculated the distributions by interchanging the order of calculating the trace and of the integration over the test functions. Our exact result is particularly interesting for the representations with $P^{2}=0$. The restriction to $A_{0}$ is the reason why the arbitrary constant $C$ in $(3.29)$ does not contribute to the distribution $\chi_{\mathrm{II}}(f)$. Because we must restrict $\chi_{\mathrm{II}}(f)$ to $A_{0}$, our result does not contradict the statement of E. Nelson and W. F. Stinnespring [16] that $\overline{\mathfrak{P}}$ is not $C C R[17]$.

The formula (4.37) gives also an explicite expression of the character of the representation $P^{2}=0$ and continuous spin:

$$
\begin{aligned}
& \chi_{\mathrm{II}}(f)=\int 2 \pi J_{0}(Q|u|) \tilde{f}\left(t^{-1}(p) \gamma(0, u) t(p), \varepsilon_{2} p\right) d u_{1} d u_{2} d \mu(p)+ \\
& +(-1)^{\tau} \int 2 \pi J_{0}(Q|u|) \tilde{f}\left(t^{-1}(p) \gamma(2 \pi, u) t(p), \varepsilon_{2} p\right) d u, d u_{2} d \mu(p) .
\end{aligned}
$$

\section{Final Remarks}

In the preceding chapters we have shown that it is possible to calculate the traces of the irreducible unitary representations of the Poincaré group, i.e. the primitive characters of this group, and it is possible to give the resulting expressions a precise mathematical meaning. The result is formulated in our theorem. This result demonstrates, for the example of the Poincaré group, the possibility to generalize Frobenius' definition of induced characters to non-compact, non-semisimple groups with the help of distribution theoretic methods. But the choice of the space on which the characters may be defined as distributions is not general. One has to take into account the special structure of the representations. This is shown by the example of the representations with 
$P_{\mu} P^{\mu}=0$. In this connection it is interesting to consider also the characters of the irreducible representations of $\overline{\mathfrak{P}}$ with $P_{\mu} \equiv 0$. These unfaithful irreducible representations of $\overline{\mathfrak{P}}$ are faithful irreducible representations of the factor group $\overline{\mathfrak{P}} / \mathfrak{T} \cong S L(2, \mathbb{C})$. Their characters [3] define distributions $\chi^{S L(2, \mathbb{C})}$ on the space $\mathscr{D}(S L(2, \mathbb{C}))$ of the infinitely often differentiable functions on $S L(2, \mathbb{C})$ with compact support. In order to define $\chi^{S L(2, \mathbb{C})}$ as a distribution on $A$ we use the mapping $I$ which maps $A \rightarrow \mathscr{D}(S L(2 C))$

$$
I: I(f(\alpha, a))=\int f(\alpha, a) d^{4} a .
$$

This mapping is continuous and satisfies

$$
I\left(f \circ f^{\prime}\right)=I(f) \circ I\left(f^{\prime}\right), \quad I\left(f^{*}\right)=(I(f))^{*}
$$

where the r.h.s. refer to the convolution and involution in $\mathscr{D}(S L(2, \mathbb{C}))$. We put

$$
\chi(f)=\chi^{S L(2, \mathbb{C})}(I f) .
$$

Because of (5.1) and (5.2), $\chi(f)$ is a real, positive, central distribution on $A$. In this form we may consider it as the character of the unfaithful representations of $\overline{\mathfrak{P}}$. It has the general form of an induced character corresponding to Eq. (4.37).

The primitive characters of $\overline{\mathfrak{P}}$ which are expressable in classical functions like Besselfunctions etc. satisfy certain completeness and orthogonality relations on the class functions $[4 \mathrm{~b}, \mathrm{~d}]$. Because of the complicated structure of the conjugation classes of $\overline{\mathfrak{P}}$ and of the different types of irreducible representations, a complete discussion of the completeness and orthogonality relations is rather involved. We shall not discuss this subject here.

We shall also not pursue the question of possible applications to physics of the primitive characters of $\overline{\mathfrak{P}}$. There are some applications to the statistical theory of elementary particle reactions [18] which takes into account the fundamental conservation laws in the framework of relativistic invariance.

We wish to thank Drs. G. Fuchs, J. Lascoux and P. Renouard for critical remarks and valuable discussions.

\section{Appendix A}

We calculate the characters of the two-dimensional euclidean group. The one-dimensional unfaithful representations are identical with their characters. The infinite dimensional irreducible representations are defined in Eq. (3.20). An orthonormal basis in the representation space is given by

$$
\Phi_{n}(\nu)=\exp (i n v), \quad n=0, \pm 1, \pm 2, \ldots .
$$


We put

$$
\text { Trace } U(\gamma)=\sum_{n=-\infty}^{n=+\infty}\left(\Phi_{n}, U(\gamma) \Phi_{n}\right)
$$

and perform the following explicite calculation in which we interchange freely integration and summation:

$$
\begin{aligned}
\operatorname{Trace} U(\gamma) & =\frac{1}{2 \pi} \int_{0}^{2 \pi} d \nu \exp \left(i Q\left(\operatorname{Re} e^{i v} u\right)\right)(-1)^{\tau|\varphi, v|} \sum_{n} \exp (i n(\varphi-2 \pi|\varphi, v|) \\
& =\left(\delta(\varphi)+(-1)^{\tau} \delta(\varphi-2 \pi)\right) \int_{0}^{2 \pi} \exp \left(i Q \cdot \operatorname{Re}\left(e^{i v} u\right)\right) d v \\
& =\left(\delta(\varphi)+(-1)^{\tau} \delta(\varphi-2 \pi)\right) \cdot 2 \pi \cdot J_{0}(Q|u|)
\end{aligned}
$$

\section{Appendix B}

1. We shall prove that the set $r(p) K r^{-1}\left(p^{\prime}\right) \cap S_{\beta}$ is bounded if $p$ and $p^{\prime}$ vary over $\mathfrak{V}_{3}$. The following is an equivalent statement: The set of those $\beta \in S_{\beta}$ is bounded for which is $r^{-1}(p) \beta r\left(p^{\prime}\right) \subset K$ for some $\left(p, p^{\prime}\right)$. For the proof it is sufficient to consider only the action of the noncompact part $a_{0}+a \sigma_{3}$ of $r(p)$ and $r\left(p^{\prime}\right)$ on $S_{\beta}$. From

$$
\left(\begin{array}{ll}
a & 0 \\
0 & a^{-1}
\end{array}\right)\left(\begin{array}{ll}
x & y \\
\bar{y} & \bar{x}
\end{array}\right)\left(\begin{array}{cc}
a^{\prime} & 0 \\
0 & a^{\prime-1}
\end{array}\right)=\left(\begin{array}{cc}
a a^{\prime} x & a a^{\prime-1} y \\
a^{-1} a^{\prime} \bar{y} & a^{-1} a^{\prime-1} \bar{x}
\end{array}\right) \in K
$$

i.e. $\left|a a^{\prime} x\right|<M(K), \quad\left|\left(a a^{\prime}\right)^{-1} \bar{x}\right|<M(K)$ etc. it follows $|x \bar{x}|<M\left(K^{2}\right)$, $|y \bar{y}|<M(K)^{2}$. This proves our statement.

2. In an analogous way we prove statement (4.31a). We have to consider only the non-compact part $\left(\begin{array}{cc}\left|\mathbf{p}^{\prime}\right|^{-1 / 2} & 0 \\ 0 & \left|\mathbf{p}^{\prime}\right|^{1 / 2}\end{array}\right)$ of $t(p)$

$$
\begin{aligned}
& \left(\begin{array}{cc}
|\mathbf{p}|^{1 / 2} & 0 \\
0 & |\mathbf{p}|^{-1 / 2}
\end{array}\right)\left(\begin{array}{ccc}
e^{i \varphi / 2} & e^{-i \varphi / 2} & \left(u_{1}+i u_{2}\right) \\
0 & e^{-i \varphi / 2} &
\end{array}\right)\left(\begin{array}{cc}
\left|\mathbf{p}^{\prime}\right|^{-1 / 2} & 0 \\
0 & \left|\mathbf{p}^{\prime}\right|^{1 / 2}
\end{array}\right) \\
& =\left(\begin{array}{ccc}
e^{i \varphi / 2}|\mathbf{p}|^{1 / 2}\left|\mathbf{p}^{\prime}\right|^{-1 / 2} & e^{-i \varphi / 2}|\mathbf{p}|^{1 / 2}\left|\mathbf{p}^{\prime}\right|^{1 / 2}\left(u_{1}+i u_{2}\right) \\
0 & e^{-i \varphi / 2}|\mathbf{p}|^{-1 / 2}\left|\mathbf{p}^{\prime}\right|^{1 / 2}
\end{array}\right) \in K^{\prime} .
\end{aligned}
$$

It follows $|\mathbf{p}|^{1 / 2}\left|\mathbf{p}^{\prime}\right|^{1 / 2}\left|u_{1}+i u_{2}\right|<M(K)$ and therefore

$$
\int_{S_{\gamma} \cap t(p) K t^{-1}\left(p^{\prime}\right)} d u_{1} d u_{2} \leqq \pi \frac{M(K)^{2}}{|\mathbf{p}|\left|\mathbf{p}^{\prime}\right|} .
$$

From the boundedness of the diagonal terms of the r.h.s. of (C.1) follows that for fixed $\left|\mathbf{p}^{\prime}\right|$ and sufficiently large $|\mathbf{p}|$ or sufficiently small $|\mathbf{p}|$ : $t(p) K t^{-1}\left(p^{\prime}\right) \cap S_{\gamma}=\theta$. This is also the case for fixed $|\mathbf{p}|$ and sufficiently large or small $\left|\mathbf{p}^{\prime}\right|$.

3. We prove now some estimates on derivatives of the test functions $\hat{f}_{p p}(s)$ defined in Eq. (4.32). 
(I) $p^{2}=1, \quad p_{0}>0$.

$$
\sup _{\substack{\varrho \in S U(2) \\ p \in \mathfrak{M}_{1}}}\left(\mid \frac{\partial^{n}}{\partial \varrho^{n}} \hat{f}_{p p}(\varrho)\left(1+|p|^{2}\right)^{m}\right)<\infty \quad \text { for all } m, n .
$$

Proof. We put

$\sup _{\mathscr{J}}\left(\left|\frac{\partial{ }^{n j_{1}}}{\partial \varrho^{n j_{1}}} \alpha_{j_{1}}\left(h^{-1}(p) \varrho h(p)\right) \cdot \ldots \cdot \frac{\partial^{n} j_{K}}{\partial \varrho^{n_{j_{K}}}} \alpha_{j_{K}}\left(h^{-1}(p) \varrho h(p)\right)\right|\right)=N_{n}(p)$.

The notation is as in the proof of lemma 3. The index $m$ stands for the conditions: $0 \leqq \sum_{i=1}^{K} n_{j_{i}} \leqq n, 1 \leqq j_{1} \leqq \cdots \leqq j_{K} \leqq 6$. Because of the algebraic form of $h^{-1}(p) \varrho h(p)$, the upper limit $N_{n}(p)$ is bounded by a sufficient large power of $\left(1+|p|^{2}\right)$ for large $|p|$. We have

$$
\sup _{\varrho \in S U(2)}\left(\left|\frac{\partial^{n}}{\partial \varrho^{n}} f_{p p} f(\varrho)\right|\right) \leqq 6^{n} n ! N_{n}^{\prime} \sup _{\alpha \in S L(2, \mathbb{C})}\left(\left|\frac{\partial^{n}}{\partial \alpha^{n}} \tilde{f}\left(\alpha, \varepsilon_{1} M p\right)\right|\right)
$$

and our statement follows from the behaviour of $f$ for large $|p|$.

Similarly we prove in the other cases

(III) $p^{2}=-1$

$$
\sup _{\substack{\beta \in K^{\prime} \\ p \in \mathfrak{V}_{3}}}\left(\left|\frac{\partial^{n}}{\partial \beta^{n}} \hat{f}_{p p}(\beta)\right|\left(1+|p|^{2}\right)^{m}\right)<\infty \text { for all } m, n
$$

$K^{\prime}$ denotes a compact set which contains the supports of $f_{p p}(\beta)$ for each $p: \bigcup_{p} r(p) K r^{-1}(p) \cap S_{\beta} \subset K^{\prime} ; K$ is the support of $\tilde{f}(\alpha, p)$ with respect to $\alpha$ in $S L(2, \mathbb{C})$. We have shown in B1 that such an $K^{\prime}$ exists.

(II) $p^{2}=0 . \varepsilon\left(p_{0}\right)=\varepsilon_{2}$.

$$
\begin{aligned}
& \sup _{\substack{\gamma \in K^{\prime} \\
p \in \mathfrak{V}_{2}}}\left(\left|\frac{\partial^{n}}{\partial \gamma^{n}} \hat{f}_{p p}(\gamma)\right|\left(1+|p|^{2}\right)^{m}\right)<\infty \text { for all } m, n \\
& K^{\prime}=\bigcup_{p \in N_{\varepsilon}} S_{\gamma} \cap t(p) K t^{-1}(p), \quad N_{\varepsilon}=\{p|| \mathbf{p} \mid>\varepsilon\} .
\end{aligned}
$$

\section{References}

1. Wigner, E. P.: Ann. Math. 40, 149 (1939).

2. Pontruagin, W.: Topological groups. Princeton: Princeton University Press 1939.

3. a) Gelfand, I. M., and M. A. Neunark: Unitäre Darstellungen der klassischen Gruppen. Berlin: Akademie-Verlag 1957. - b) Neumark, M. A.: Lineare Darstellungen der Lorentzgruppe. Berlin: VEB-Verlag der Wissenschaften 1963.

4. a) Schrader, R.: Diplomarbeit Hamburg 1964. - b) Joos, H., and R. SchraDER: Boulder Symposium on the Lorentzgroup. Boulder University of Colorado Press 1964. This preliminary report is now superseded by the present paper. - c) Fuchs, G., and P. RenouaRd: E.N.S. preprints 1966 and 1967. - d) RüHL, W.: Rockefeller University preprint 1967.

4 Commun. math. Phys., Vol. 7 
5. Bargmann, V.: Ann. Math. 48, 568 (1947).

6. Mackey, G. W.: University of Chicago mimiographed notes (1955). Acta Math. 99, 265 (1958).

7. Frobenius, G.: Sitzber. Königl. Preuss. Akad. 501 (1898).

8. V. D. WaErden, B. L.: Die gruppentheoretischen Methoden in der Quantenmechanik. Berlin: Springer 1932.

9. Wightman, A. S.: Lecture notes Cargèse Corsica 1964.

10. Erdelyi, A.: Higher transcendental functions. New York: Mc Graw-Hill 1953.

11. JACOB, M., and G. C. WICK: Ann. Phys. 7, 404 (1959).

12. a) Harish-Chandra: Proc. Nat. Acad. Sci. 38, 337 (1952). - b) Gelfand, I. M., and Geraev, I.: Uspheki 18 (4), 29 (1963).

13. Curtis, C. W., and I. Reiner: Representation theory of finite groups and associative algebras. New York-London: Interscience 1962.

14. Schwartz, L.: Theorie des distributions I, II. Paris: Hermann 1957/59.

15. Kӧтне, G.: Topologische lineare Räume. Berlin-Göttingen-Heidelberg: Springer 1960.

16. Nelson, E., and W. F. Stinnespring: Ann. Math. 81, 547 (1959).

17. Kaplansky, L.: Trans. Am. Math. Soc. 70, 219 (1951).

18. Satz, H.: Habilitationsschrift Hamburg 1967. 